\title{
On approximation properties of generalized Lupaş type operators based on Polya distribution with Pochhammer $k$-symbol
}

\author{
Övgü Gürel Yılmaz ${ }^{1}$ (D), Rabia Aktaş² (D), Fatma Taşdelen² (D), Ali Olgun³ (D) \\ ${ }^{1}$ Recep Tayyip Erdogan University, Department of Mathematics, 53100 Rize, Turkey \\ ${ }^{2}$ Ankara University, Faculty of Science, Department of Mathematics, 06100, Tandoğan, Ankara, Turkey \\ ${ }^{3}$ Kırıkkale University, Department of Mathematics, 71450 Yahşihan, Kırıkkale, Turkey
}

\begin{abstract}
The purpose of this paper is to introduce a Kantorovich variant of Lupas-Stancu operators based on Polya distribution with Pochhammer $k$-symbol. We obtain rates of convergence for these operators by means of the classical modulus of continuity. Also, we give a Voronovskaja type theorem for the pointwise approximation. Furthermore, we construct a bivariate generalization of these operators and we discuss some convergence properties of them. Finally, we present some figures to compare approximation properties of our new operators with those of other operators which are mentioned in this paper. We observe that the approximation of our operators to the function $f$ is better than that of some other operators in a certain range of values of $k$.
\end{abstract}

Mathematics Subject Classification (2020). 26A16, 41A10, 41A25, 41A36

Keywords. Bernstein operators, Stancu operators, Lupaş operators, Kantorovich operators, Polya distribution, modulus of continuity, Lipschitz class, Voronovskaja type theorem, Pochhammer $k$-symbol

\section{Introduction}

In the field of approximation theory, Bernstein operators have considerable importance in the proof of the Weierstrass approximation theorem. In 1912, Bernstein [9] presented the well-known Bernstein operators in the following form

$$
B_{n}(f ; x)=\sum_{m=0}^{n}\left(\begin{array}{c}
n \\
m
\end{array}\right) x^{m}(1-x)^{n-m} f\left(\frac{m}{n}\right), f \in C[0,1],
$$

where $C[0,1]$ is the space of all real-valued continuous functions on $[0,1]$. Since they are functional in studying many problems and convenient in computer-aided studies, they have important generalizations and applications. We refer to $[1,2,23,28,29,32]$.

\footnotetext{
*Corresponding Author.

Email addresses: ovgu.gurelyilmaz@erdogan.edu.tr (Ö. Gürel Yılmaz), raktas@science.ankara.edu.tr (R. Aktaş), tasdelen@science.ankara.edu.tr (F. Taşdelen), aolgun@kku.edu.tr (A. Olgun)

Received: 08.04.2021; Accepted: 25.09.2021
} 
In 1968, Stancu [35] introduced the operators $P_{n}^{\langle\alpha\rangle}: C[0,1] \rightarrow C[0,1]$

$$
P_{n}^{\langle\alpha\rangle}(f ; x)=\sum_{m=0}^{n} p_{n, m}^{\langle\alpha\rangle}(x) f\left(\frac{m}{n}\right),
$$

with a nonnegative parameter $\alpha$ where $p_{n, m}^{\langle\alpha\rangle}$ is defined by

$$
p_{n, m}^{\langle\alpha\rangle}(x)=\left(\begin{array}{c}
n \\
m
\end{array}\right) \frac{\prod_{\nu=0}^{m-1}(x+\nu \alpha) \prod_{\mu=0}^{n-m-1}(1-x+\mu \alpha)}{(1+\alpha)(1+2 \alpha) \ldots(1+(n-1) \alpha)},
$$

for $n \in \mathbb{N}$. It is worthy of note that for $\alpha=0$, the operators (1.2) lead to the classical Bernstein operators given by (1.1).

After Stancu's paper, by taking into account the special choice $\alpha=\frac{1}{n}$ in (1.2), Lupaş and Lupaş [19] constructed the operators $P_{n}^{\left\langle\frac{1}{n}\right\rangle}: C[0,1] \rightarrow C[0,1]$ as follows

$$
P_{n}^{\left\langle\frac{1}{n}\right\rangle}(f ; x)=\frac{2 n !}{(2 n) !} \sum_{m=0}^{n}\left(\begin{array}{c}
n \\
m
\end{array}\right)(n x)_{m}(n-n x)_{n-m} f\left(\frac{m}{n}\right),
$$

where $(s)_{m}$ is a rising factorial, also known as the Pochhammer symbol, namely

$$
(s)_{m}=\left\{\begin{aligned}
s(s+1)(s+2) \ldots(s+m-1) & \text { for } m \in \mathbb{N} \\
1 & \text { for } m=0, s \neq 0
\end{aligned}\right.
$$

where $s$ is a real or complex number.

In 1989, Razi [33] defined the Kantorovich modification of the Bernstein-Stancu operators $P_{n}^{\langle\alpha\rangle}(f ; x)$ as follows

$$
K_{n}^{(\alpha)}(f ; x)=(n+1) \sum_{m=0}^{n} p_{n, m}^{\langle\alpha\rangle}(x) \int_{\frac{m}{n+1}}^{\frac{m+1}{n+1}} f(t) d t
$$

and studied some approximation properties. For $\alpha=0$, these operators return to the classical Bernstein Kantorovich operators

$$
K_{n}(f ; x)=(n+1) \sum_{m=0}^{n}\left(\begin{array}{c}
n \\
m
\end{array}\right) x^{m}(1-x)^{n-m} \int_{\frac{m}{n+1}}^{\frac{m+1}{n+1}} f(t) d t .
$$

In 2012, Miclăuş [20] reconsidered the operators given by (1.4) and recalculated some of the properties such as moments, the remainder term and the monotocity of the operators with a different technique. Also, asymptotic behaviour of the operators (1.4) was discussed.

In 2016, for $\alpha=\frac{1}{n}$, the Kantorovich modification of Lupaş operators $P_{n}^{\left\langle\frac{1}{n}\right\rangle}(f ; x)$ based on Polya distribution was studied by Agrawal et al. [6] as follows

$$
\begin{aligned}
D_{n}^{*\left(\frac{1}{n}\right)}(f ; x) & =(n+1) \frac{2 n !}{(2 n) !} \sum_{m=0}^{n}\left(\begin{array}{c}
n \\
m
\end{array}\right)(n x)_{m}(n-n x)_{n-m} \\
& \times \int_{\frac{m}{n+1}}^{\frac{m+1}{n+1}} f(t) d t, \quad f \in C[0,1],
\end{aligned}
$$


local and global approximation properties were obtained. Furthermore, the authors introduced the bivariate form of the operators (1.7) as follows

$$
\begin{aligned}
D_{n_{1}, n_{2}}^{*\left(\frac{1}{n_{1}}, \frac{1}{n_{2}}\right)}(f ; x, y)= & \left(n_{1}+1\right)\left(n_{2}+1\right) \sum_{m_{1}=0}^{n_{1}} \sum_{m_{2}=0}^{n_{2}} p_{n_{1}, n_{2}, m_{1}, m_{2}}^{\left(1 / n_{1}, 1 / n_{2}\right)}(x, y) \\
& \times \int_{\frac{m_{1}}{n_{1}+1}}^{\frac{m_{1}+1}{n_{1}+1}} \int_{\frac{m_{2}}{n_{2}+1}}^{\frac{m_{2}+1}{n_{2}+1}} f(t, s) d t d s,
\end{aligned}
$$

for $f: C\left(J^{2}\right) \rightarrow C\left(J^{2}\right), J=[0,1]$ where

$$
\begin{aligned}
p_{n_{1}, n_{2}, m_{1}, m_{2}}^{\left(1 / n_{1}, 1 / n_{2}\right)}(x, y) & =\frac{2 n_{1} !}{\left(2 n_{1}\right) !} \frac{2 n_{2} !}{\left(2 n_{2}\right) !}\left(\begin{array}{c}
n_{1} \\
m_{1}
\end{array}\right)\left(\begin{array}{c}
n_{2} \\
m_{2}
\end{array}\right)\left(n_{1} x\right)_{m_{1}} \\
& \times\left(n_{1}-n_{1} x\right)_{n_{1}-m_{1}}\left(n_{2} y\right)_{m_{2}}\left(n_{2}-n_{2} y\right)_{n_{2}-m_{2}}
\end{aligned}
$$

and they gave some rates of convergence for these operators.

Recently, many authors have studied new applications of the linear positive operators based on Polya distribution. We refer to [3-5, 10,11,13-15,21, 22, 26, 31].

In 2007, the notion of Pochhammer $k$-symbol was first proposed by Diaz and Pariguan in [12]. For $\lambda \in \mathbb{C}$, it is defined by

$$
(\lambda)_{m, k}=\left\{\begin{array}{cc}
\lambda(\lambda+k)(\lambda+2 k) \ldots(\lambda+(m-1) k) & ; \quad m \geq 1 \\
1 \quad ; & m=0, \lambda \neq 0
\end{array}\right.
$$

where $m \in \mathbb{N}$ and $k$ is a nonnegative real number. It is easy to see that for $k=1$, the definition of Pochhammer $k$-symbol coincides with the usual Pochhammer symbol which is given by (1.5). This investigation has revealed many new generalizations such as $k$ Gamma function, $k$-Beta function, $k$-Zeta function, $k$-generalization of hypergeometric function and so on. For more details, see [12,16-18,24, 25].

Our present study is essentially motivated by the results based on Polya distribution in [19] and [20], and the Pochhammer $k$-symbol given by [12]. We define a slight modification of Polya distribution for the special case $\alpha=\frac{k}{n}$ where $k$ is a nonnegative real number by applying the notion of Pochhammer $k$-symbol. The main objective of this paper is to investigate the rates of convergence of the operators.

The structure of the paper is as follows. In section 2, we first consider a Lupaş type generalization $P_{n, k}^{\left\langle\frac{k}{n}\right\rangle}(f ; x)$ for the case $\alpha=\frac{k}{n}$ of the Bernstein-Stancu operators $P_{n}^{\langle\alpha\rangle}(f ; x)$. We compare the convergence of the operators $P_{n, k}^{\left\langle\frac{k}{n}\right\rangle}(f ; x)$ with that of the operators $P_{n}^{\left\langle\frac{1}{n}\right\rangle}(f ; x)$ using some graphics. We show that for $0 \leq k \leq 1$ the operators $P_{n, k}^{\left\langle\frac{k}{n}\right\rangle}(f ; x)$ give a better approximation than $P_{n}^{\left\langle\frac{1}{n}\right\rangle}(f ; x)$. Then we introduce a Kantorovich-Stancu modification of the Lupaş type operators $P_{n, k}^{\left\langle\frac{k}{n}\right\rangle}$ with Pochhammer $k$-symbol and present some approximation properties of these operators. More precisely, we estimate the rates of the convergence by means of the classical modulus of continuity and discuss pointwise approximation via Voronovskaja type theorem. Section 3 presents a bivariate generalization of KantorovichStancu modification of the Lupaş type operators $P_{n, k}^{\left\langle\frac{k}{n}\right\rangle}$. Some preliminary results for the bivariate operators are given and some approximation properties are discussed. Finally, taking into account some illustrative graphics which are obtained with the help of Maple software, we compare the rates of convergence of our bivariate operators with those of some other operators for the different values of the parameter $k$. 


\section{Lupaş type operators by means of Pochhammer $k$-symbol}

Before starting this section, we briefly describe the Lupas type operators that play an important role in this paper. Let $C[0,1]$ be the space of all real valued continuous functions on $[0,1]$ endowed with the norm

$$
\|f\|_{C[0,1]}=\sup _{x \in[0,1]}|f(x)| .
$$

Let $n \in \mathbb{N}$ and $k$ be a nonnegative real number. By taking into account the special choice $\alpha=\frac{k}{n}$ in (1.2), the operators $P_{n, k}^{\left\langle\frac{k}{n}\right\rangle}: C[0,1] \rightarrow C[0,1]$ are defined by

$$
P_{n, k}^{\left\langle\frac{k}{n}\right\rangle}(f ; x)=\frac{1}{(n)_{n, k}} \sum_{m=0}^{n}\left(\begin{array}{c}
n \\
m
\end{array}\right)(n x)_{m, k}(n-n x)_{n-m, k} f\left(\frac{m}{n}\right)
$$

where $(\lambda)_{m, k}$ is a Pochhammer $k$-symbol given by (1.9). For the case $k=0$, the operators (2.1) turn to the classical Bernstein operators. For $k=1$, the operators (2.1) reduce to the Lupaş operators given by (1.4). In this paper, we deal with the operators $P_{n, k}^{\left\langle\frac{k}{n}\right\rangle}(f ; x)$ for the case $k>0$.

Now, we begin our study by giving moments, central moments and rate of convergence for the operators (2.1). We first give Lemma 2.1, Lemma 2.3 and Corollary 2.2 without proof, which follow from the results given in the paper [20] for $\alpha=\frac{k}{n}, k>0$.

Throughout this paper, let us denote the monomials $e_{j}(t)=t^{j}$ for $j \in \mathbb{N}_{0}$ where $\mathbb{N}$ is the set of positive integers and $\mathbb{N}_{0}=\mathbb{N} \cup\{0\}$.

Lemma 2.1. Let $n \in \mathbb{N}$ and $k>0$. Then for the operators $P_{n, k}^{\left\langle\frac{k}{n}\right\rangle}$ defined by (2.1), we have

$$
\begin{aligned}
P_{n, k}^{\left\langle\frac{k}{n}\right\rangle}\left(e_{0} ; x\right) & =1, \\
P_{n, k}^{\left\langle\frac{k}{n}\right\rangle}\left(e_{1} ; x\right) & =x, \\
P_{n, k}^{\left\langle\frac{k}{n}\right\rangle}\left(e_{2} ; x\right) & =x^{2}+\frac{(k+1) x(1-x)}{n+k}, \\
P_{n, k}^{\left\langle\frac{k}{n}\right\rangle}\left(e_{3} ; x\right) & =x^{3}+\frac{(3 n+2 k-2)(k+1) x^{2}(1-x)}{(n+k)(n+2 k)} \\
& +\frac{(2 k+1)(k+1) x(1-x)}{(n+k)(n+2 k)}, \\
P_{n, k}^{\left\langle\frac{k}{n}\right\rangle}\left(e_{4} ; x\right) & =x^{4}+\frac{(k+1)\left((11 n-6)(k-1)+6\left(n^{2}+k^{2}\right)\right) x^{3}(1-x)}{(n+k)(n+2 k)(n+3 k)} \\
& +\frac{(k+1)\left(7 n+11 n k+6\left(k^{2}-k-1\right)\right) x^{2}(1-x)}{(n+k)(n+2 k)(n+3 k)} \\
& +\frac{(k+1)(n-k+6 n k(k+1)) x(1-x)}{n(n+k)(n+2 k)(n+3 k)} .
\end{aligned}
$$


Corollary 2.2. Let $n \in \mathbb{N}$ and $k>0$. Then the central moments of the operators $P_{n, k}^{\left\langle\frac{k}{n}\right\rangle}$ are given by

$$
\begin{aligned}
P_{n, k}^{\left\langle\frac{k}{n}\right\rangle}\left(\left(e_{1}-x\right)^{2} ; x\right) & =\frac{(k+1) x(1-x)}{n+k}, \\
P_{n, k}^{\left\langle\frac{k}{n}\right\rangle}\left(\left(e_{1}-x\right)^{3} ; x\right) & =\frac{(k+1)(2 k+1) x(1-x)(1-2 x)}{(n+k)(n+2 k)}, \\
P_{n, k}^{\left\langle\frac{k}{n}\right\rangle}\left(\left(e_{1}-x\right)^{4} ; x\right) & =\frac{(k+1) 3 n(-2+n+k(-6-6 k+n))(x(1-x))^{2}}{n(n+k)(n+2 k)(n+3 k)} \\
& +\frac{(k+1)(n+k(-1+6(k+1) n)) x(1-x)}{n(n+k)(n+2 k)(n+3 k)} .
\end{aligned}
$$

Lemma 2.3. Let $n \in \mathbb{N}$ and $k>0$. Then for every $f \in C[0,1]$, we have

uniformly in $[0,1]$.

$$
\lim _{n \rightarrow \infty} P_{n, k}^{\left\langle\frac{k}{n}\right\rangle}(f ; x)=f(x)
$$

For $\delta>0$, the modulus of continuity of $f$ denoted by $w(f ; \delta)$ is defined by

$$
\omega(f ; \delta)=\sup _{\substack{x, t \in[a, b] \\|t-x| \leq \delta}}|f(t)-f(x)| .
$$

Then, for any $\delta>0$ and each $x \in[a, b]$, it is well known that

$$
|f(t)-f(x)| \leq\left(\frac{|t-x|}{\delta}+1\right) \omega(f ; \delta) .
$$

For $\alpha=\frac{k}{n}, k>0$, we can give the next theorem from the results given in [32].

Theorem 2.4. Let $n \in \mathbb{N}$ and $k>0$. Then for every $f \in C[0,1]$, the following inequality holds

$$
\left\|P_{n, k}^{\left\langle\frac{k}{n}\right\rangle}(f ; x)-f(x)\right\|_{C[0,1]} \leq \frac{3}{2} \omega\left(f, \sqrt{\frac{k+1}{n+k}}\right),
$$

for $k=1$, which concludes that

$$
\left\|P_{n}^{\left\langle\frac{1}{n}\right\rangle}(f ; x)-f(x)\right\|_{C[0,1]} \leq \frac{3}{2} \omega\left(f, \sqrt{\frac{2}{n+1}}\right) .
$$

Remark 2.5. Let $n \in \mathbb{N}$. Then for every $f \in C[0,1]$, the inequality

$$
P_{n, k}^{\left\langle\frac{k}{n}\right\rangle}\left(\left(e_{1}-x\right)^{2} ; x\right) \leq P_{n}^{\left\langle\frac{1}{n}\right\rangle}\left(\left(e_{1}-x\right)^{2} ; x\right)
$$

holds for $0 \leq k \leq 1$. In the case of $k=0$, the operators $P_{n, k}^{\left\langle\frac{k}{n}\right\rangle}$ become the classical Bernstein operators which give the best result concerning the approximation behaviour. For $0<k \leq 1$, the approximation of the operators $P_{n, k}^{\left\langle\frac{k}{n}\right\rangle}$ is at least as good as that of the classical operators $P_{n}^{\left\langle\frac{1}{n}\right\rangle}$.

Now, we demonstrate the behaviour of the approximation for the operators $P_{n, k}^{\left\langle\frac{k}{n}\right\rangle}$ by graphical examples.

Example 2.6. Let $f(x)=20 x^{6}+3 x^{3}-5 x^{2}+2 x, n=10$ and $k=0.1$. In Figure 1, we analyse the convergence of the operators $P_{n, k}^{\left\langle\frac{k}{n}\right\rangle}$, the classical operators $P_{n}^{\left\langle\frac{1}{n}\right\rangle}$ and the classical Bernstein operators $B_{n}$ to the function $f$. It is seen that for $k=0.1$ the operators $P_{n, k}^{\left\langle\frac{k}{n}\right\rangle}$ provide a better approximation than the operators $P_{n}^{\left\langle\frac{1}{n}\right\rangle}$. 


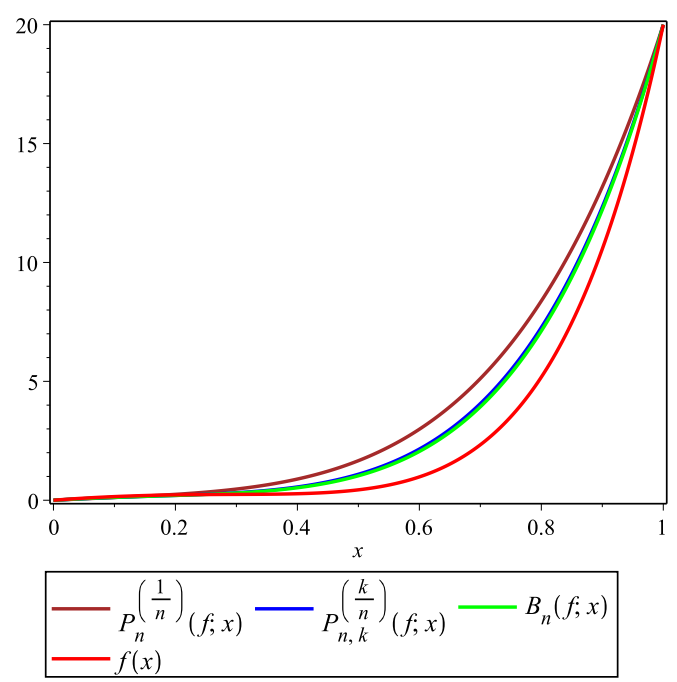

Figure 1. Convergence of $P_{n, k}^{\left\langle\frac{k}{n}\right\rangle}, P_{n}^{\left\langle\frac{1}{n}\right\rangle}$ and $B_{n}$ to the function $f$ for $n=10$ and $k=0.1$

Example 2.7. Let us consider $f(x)=\sin (6 \pi x)+5 \sin \left(\frac{1}{3} \pi x\right)$. Figure 2 illustrates the approximation process of the operators $P_{n, k}^{\left\langle\frac{k}{n}\right\rangle}$ for $k=0.5$ and the special choices of $n=$ 10,50 and 100. It can be observed that as the value of $n$ increases, the approximation of the operators $P_{n, k}^{\left\langle\frac{k}{n}\right\rangle}$ is getting better.

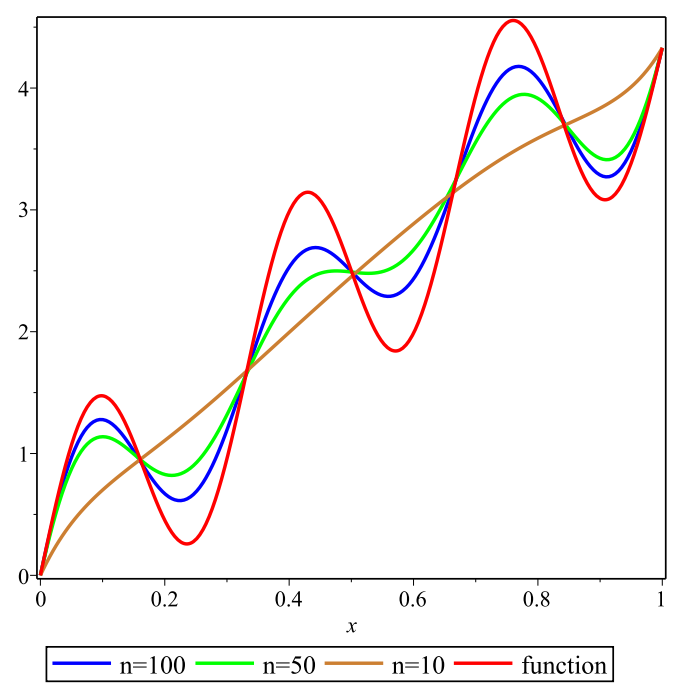

Figure 2. Approximation of the operators $P_{n, k}^{\left\langle\frac{k}{n}\right\rangle}$ for $k=0.5$ and $n=10,50,100$ 
Example 2.8. Let $f(x)=3\left(x^{2}+5 x\right) \cos \left(\frac{1}{2} \pi x\right)$. Figure 3 presents the convergence of $P_{n, k}^{\left\langle\frac{k}{n}\right\rangle}$ to the function $f$ for $n=10$ and $k=0.1,0.3,0.6,1$ and 3. From this figure, it follows that when $k$ gets smaller towards to zero, approximation is better than others.

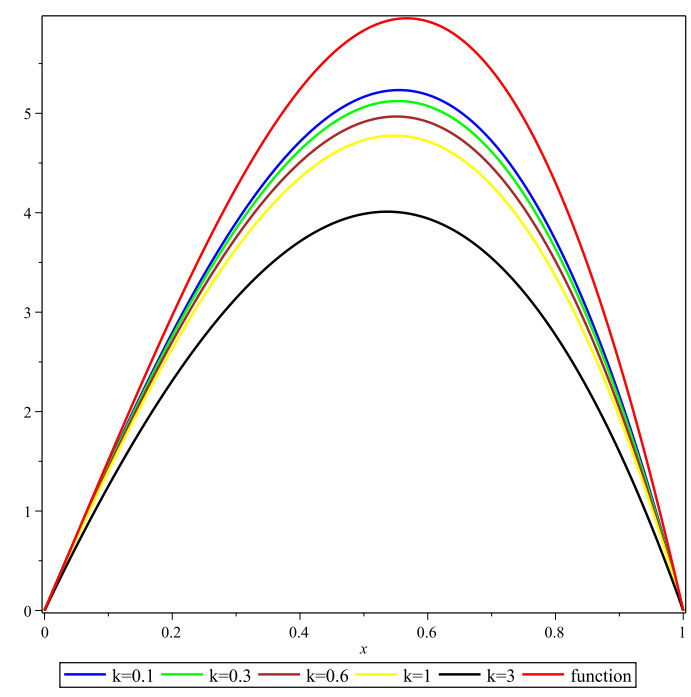

Figure 3. Approximation of the operators $P_{n, k}^{\left\langle\frac{k}{n}\right\rangle}$ for $n=10$ and $k=0.1,0.3,0.6,1,3$

Now we consider a Kantorovich-Stancu modification of Lupaş type operators (2.1) as follows

$$
K_{n}^{(\alpha, \beta, k)}(f ; x)=\frac{n+\beta+1}{(n)_{n, k}} \sum_{m=0}^{n}\left(\begin{array}{c}
n \\
m
\end{array}\right)(n x)_{m, k}(n-n x)_{n-m, k} \int_{\frac{m+\alpha}{n+\beta+1}}^{\frac{m+\alpha+1}{n+\beta+1}} f(t) d t
$$

where $\alpha, \beta, k$ are nonnegative real numbers and $0 \leq \alpha \leq \beta$. In the case of $k=0$, the operators (2.5) reduce to the Kantorovich-Stancu modification of Bernstein operators [8]. For $k=1,(2.5)$ gives the special case $\alpha_{2}=\beta_{2}=0$ of Kantorovich type Lupas-Stancu operators given in [31]. For $k=1$ and $\alpha=\beta=0$, these operators lead to the Kantorovich modification of the operators $P_{n}^{\left\langle\frac{1}{n}\right\rangle}(f ; x)$ given by $(1.7)$.

In this section, we deal with the approximation properties of the operators $K_{n}^{(\alpha, \beta, k)}$ for the case $k>0$. Taking into account the results given for the operators $P_{n, k}^{\left\langle\frac{k}{n}\right\rangle}$ in Lemma 2.1 , we can give the next lemma.

Lemma 2.9. Let $n \in \mathbb{N}, k>0$. Then for the operators $K_{n}^{(\alpha, \beta, k)}$ defined by (2.5), we have 


$$
\begin{aligned}
& K_{n}^{(\alpha, \beta, k)}\left(e_{0} ; x\right)=1, \\
& K_{n}^{(\alpha, \beta, k)}\left(e_{1} ; x\right)=\frac{n x}{n+\beta+1}+\frac{2 \alpha+1}{2(n+\beta+1)}, \\
& K_{n}^{(\alpha, \beta, k)}\left(e_{2} ; x\right)=\frac{n^{2}}{(n+\beta+1)^{2}}\left\{x^{2}+\frac{k+1}{n+k} x(1-x)\right\}+\frac{(2 \alpha+1) n}{(n+\beta+1)^{2}} x+\frac{(\alpha+1)^{3}-\alpha^{3}}{3(n+\beta+1)^{2}}, \\
& K_{n}^{(\alpha, \beta, k)}\left(e_{3} ; x\right)=\frac{(n-1)(n-2) n^{3} x^{3}}{(n+\beta+1)^{3}(n+k)(n+2 k)}+\frac{3 n^{2}(n-1)((3+2 \alpha+2 k) n+2(1+2 \alpha) k) x^{2}}{2(n+\beta+1)^{3}(n+k)(n+2 k)} \\
& +\left(\frac{4(1+3 \alpha(1+\alpha)) k^{2} n+6 k(2+k+\alpha(5+3 \alpha+2 k)) n^{2}}{2(n+\beta+1)^{3}(n+k)(n+2 k)}\right. \\
& \left.+\frac{\left(7+6 \alpha^{2}+6 \alpha(2+k)+k(9+4 k)\right) n^{3}}{2(n+\beta+1)^{3}(n+k)(n+2 k)}\right) x+\frac{4 \alpha^{3}+6 \alpha^{2}+4 \alpha+1}{4(n+\beta+1)^{3}}, \\
& K_{n}^{(\alpha, \beta, k)}\left(e_{4} ; x\right)=\frac{(n-1)(n-2)(n-3) n^{4} x^{4}}{(n+\beta+1)^{4}(n+k)(n+2 k)(n+3 k)} \\
& +\frac{2 n^{3}(n-1)(n-2)((4+2 \alpha+3 k) n+3(1+2 a) k) x^{3}}{(n+\beta+1)^{4}(n+k)(n+2 k)(n+3 k)} \\
& +\left(\frac{-12(1+3 \alpha(1+\alpha)) k^{2} n^{2}+k(-27-5 k+6 \alpha(-11+\alpha(-5+6 k))) n^{3}}{(n+\beta+1)^{4}(n+k)(n+2 k)(n+3 k)}\right. \\
& +\frac{3\left(-5-2 \alpha(3+\alpha)+k+2 \alpha(9+5 \alpha) k+2(1+6 \alpha) k^{2}\right) n^{4}}{(n+\beta+1)^{4}(n+k)(n+2 k)(n+3 k)} \\
& \left.+\frac{\left(15+6 \alpha^{2}+6 \alpha(3+2 k)+k(24+11 k)\right) n^{5}}{(n+\beta+1)^{4}(n+k)(n+2 k)(n+3 k)}\right) x^{2} \\
& +\left(\frac{6(1+2 \alpha(2+\alpha(3+2 \alpha))) k^{3} n+k^{2}(23+12 k) n^{2}}{(n+\beta+1)^{4}(n+k)(n+2 k)(n+3 k)}\right. \\
& +\frac{k^{2}\left(2 \alpha\left(40+51 \alpha+22 \alpha^{2}+18(1+\alpha) k\right)\right) n^{2}}{(n+\beta+1)^{4}(n+k)(n+2 k)(n+3 k)} \\
& +\frac{3 k\left(7+22 \alpha+22 \alpha^{2}+8 \alpha^{3}+9 k+22 \alpha k+10 \alpha^{2} k+4 k^{2}+8 \alpha k^{2}\right) n^{3}}{(n+\beta+1)^{4}(n+k)(n+2 k)(n+3 k)} \\
& \left.+\frac{\left(6+2 \alpha(7+2 \alpha(3+\alpha))+15 k+6 \alpha(3+\alpha) k+8(2+\alpha) k^{2}+6 k^{3}\right) n^{4}}{(n+\beta+1)^{4}(n+k)(n+2 k)(n+3 k)}\right) x \\
& +\frac{5 \alpha^{4}+10 \alpha^{3}+10 \alpha^{2}+5 \alpha+1}{5(n+\beta+1)^{4}} \text {. }
\end{aligned}
$$


Proof. By recalling the moments of $P_{n, k}^{\left\langle\frac{k}{n}\right\rangle}$ which are indicated in Lemma 2.1, we can easily complete the proof. For $f(t)=e_{0}(t)$, we have

$$
\begin{aligned}
K_{n}^{(\alpha, \beta, k)}\left(e_{0} ; x\right) & =\frac{n+\beta+1}{(n)_{n, k}} \sum_{m=0}^{n}\left(\begin{array}{c}
n \\
m
\end{array}\right)(n x)_{m, k}(n-n x)_{n-m, k} \int_{\frac{m+\alpha}{n+\beta+1}}^{\frac{m+\alpha+1}{n+\beta+1}} d t \\
& =\frac{n+\beta+1}{(n)_{n, k}} \sum_{m=0}^{n}\left(\begin{array}{c}
n \\
m
\end{array}\right)(n x)_{m, k}(n-n x)_{n-m, k}\left(\frac{1}{n+\beta+1}\right) \\
& =\frac{1}{(n)_{n, k}} \sum_{m=0}^{n}\left(\begin{array}{c}
n \\
m
\end{array}\right)(n x)_{m, k}(n-n x)_{n-m, k} \\
& =P_{n, k}^{\left\langle\frac{k}{n}\right\rangle}\left(e_{0} ; x\right) \\
& =1 .
\end{aligned}
$$

For the case $f(t)=e_{1}(t)$, it follows

$$
\begin{aligned}
K_{n}^{(\alpha, \beta, k)}\left(e_{1} ; x\right) & =\frac{n+\beta+1}{(n)_{n, k}} \sum_{m=0}^{n}\left(\begin{array}{c}
n \\
m
\end{array}\right)(n x)_{m, k}(n-n x)_{n-m, k} \int_{\frac{m+\alpha}{n+\beta+1}}^{\frac{m+\alpha+1}{n+\beta+1}} t d t \\
& =\frac{n+\beta+1}{2(n)_{n, k}} \sum_{m=0}^{n}\left(\begin{array}{c}
n \\
m
\end{array}\right)(n x)_{m, k}(n-n x)_{n-m, k}\left(\frac{(m+\alpha+1)^{2}-(m+\alpha)^{2}}{(n+\beta+1)^{2}}\right) \\
& =\frac{2 \alpha+1}{2(n+\beta+1)} \frac{1}{(n)_{n, k}} \sum_{m=0}^{n}\left(\begin{array}{c}
n \\
m
\end{array}\right)(n x)_{m, k}(n-n x)_{n-m, k} \\
& +\frac{n}{n+\beta+1} \frac{1}{(n)_{n, k}} \sum_{m=0}^{n}\left(\begin{array}{c}
n \\
m
\end{array}\right)(n x)_{m, k}(n-n x)_{n-m, k}\left(\frac{m}{n}\right) \\
& =\frac{n}{n+\beta+1} P_{n, k}^{\left\langle\frac{k}{n}\right\rangle}\left(e_{1} ; x\right)+\frac{2 \alpha+1}{2(n+\beta+1)} P_{n, k}^{\left\langle\frac{k}{n}\right\rangle}\left(e_{0} ; x\right) \\
& =\frac{n x}{n+\beta+1}+\frac{2 \alpha+1}{2(n+\beta+1)} .
\end{aligned}
$$

Taking into account the values of $P_{n, k}^{\left\langle\frac{k}{n}\right\rangle}\left(e_{i} ;.\right)(i=0,1,2)$, we can write

$$
\begin{aligned}
K_{n}^{(\alpha, \beta, k)}\left(e_{2} ; x\right) & =\frac{n+\beta+1}{(n)_{n, k}} \sum_{m=0}^{n}\left(\begin{array}{c}
n \\
m
\end{array}\right)(n x)_{m, k}(n-n x)_{n-m, k} \int_{\frac{m+\alpha}{n+\beta+1}}^{\frac{m+\alpha+1}{n+\beta+1}} t^{2} d t \\
& =\frac{1}{3(n+\beta+1)^{2}(n)_{n, k}} \sum_{m=0}^{n}\left(\begin{array}{c}
n \\
m
\end{array}\right)(n x)_{m, k}(n-n x)_{n-m, k}(m+\alpha+1)^{3} \\
& -\frac{1}{3(n+\beta+1)^{2}(n)_{n, k}} \sum_{m=0}^{n}\left(\begin{array}{c}
n \\
m
\end{array}\right)(n x)_{m, k}(n-n x)_{n-m, k}(m+\alpha)^{3}
\end{aligned}
$$




$$
\begin{aligned}
& =\frac{n^{2}}{(n+\beta+1)^{2}} \frac{1}{(n)_{n, k}} \sum_{m=0}^{n}\left(\begin{array}{c}
n \\
m
\end{array}\right)(n x)_{m, k}(n-n x)_{n-m, k}\left(\frac{m^{2}}{n^{2}}\right) \\
& +\frac{(2 \alpha+1) n}{(n+\beta+1)^{2}} \frac{1}{(n)_{n, k}} \sum_{m=0}^{n}\left(\begin{array}{c}
n \\
m
\end{array}\right)(n x)_{m, k}(n-n x)_{n-m, k}\left(\frac{m}{n}\right) \\
& +\frac{(\alpha+1)^{3}-\alpha^{3}}{3(n+\beta+1)^{2}} \frac{1}{(n)_{n, k}} \sum_{m=0}^{n}\left(\begin{array}{c}
n \\
m
\end{array}\right)(n x)_{m, k}(n-n x)_{n-m, k} \\
& =\frac{n^{2}}{(n+\beta+1)^{2}} P_{n, k}^{\left\langle\frac{k}{n}\right\rangle}\left(e_{2} ; x\right)+\frac{(2 \alpha+1) n}{(n+\beta+1)^{2}} P_{n, k}^{\left\langle\frac{k}{n}\right\rangle}\left(e_{1} ; x\right) \\
& +\frac{(\alpha+1)^{3}-\alpha^{3}}{3(n+\beta+1)^{2}} P_{n, k}^{\left\langle\frac{k}{n}\right\rangle}\left(e_{0} ; x\right) \\
& =\frac{n^{2}}{(n+\beta+1)^{2}}\left\{x^{2}+\frac{k+1}{n+k} x(1-x)\right\}+\frac{(2 \alpha+1) n}{(n+\beta+1)^{2}} x+\frac{(\alpha+1)^{3}-\alpha^{3}}{3(n+\beta+1)^{2}}
\end{aligned}
$$

The proof for $f(t)=e_{i}(t), i=3,4$ is quite similar to others, hence the results are given as follows

$$
\begin{aligned}
K_{n}^{(\alpha, \beta, k)}\left(e_{3} ; x\right) & =\frac{n+\beta+1}{(n)_{n, k}} \sum_{m=0}^{n}\left(\begin{array}{c}
n \\
m
\end{array}\right)(n x)_{m, k}(n-n x)_{n-m, k} \int_{\frac{m+\alpha}{n+\beta+1}}^{\frac{m+\alpha+1}{n+\beta+1}} t^{3} d t \\
& =\frac{1}{4(n+\beta+1)^{3}}\left\{4 n^{3} P_{n, k}^{\left\langle\frac{k}{n}\right\rangle}\left(e_{3} ; x\right)+6(2 \alpha+1) n^{2} P_{n, k}^{\left\langle\frac{k}{n}\right\rangle}\left(e_{2} ; x\right)\right. \\
& \left.+4\left(3 \alpha^{2}+3 \alpha+1\right) n P_{n, k}^{\left\langle\frac{k}{n}\right\rangle}\left(e_{1} ; x\right)+\left((\alpha+1)^{4}-\alpha^{4}\right) P_{n, k}^{\left\langle\frac{k}{n}\right\rangle}\left(e_{0} ; x\right)\right\}
\end{aligned}
$$

and

$$
\begin{aligned}
K_{n}^{(\alpha, \beta, k)}\left(e_{4} ; x\right) & =\frac{n+\beta+1}{(n)_{n, k}} \sum_{m=0}^{n}\left(\begin{array}{c}
n \\
m
\end{array}\right)(n x)_{m, k}(n-n x)_{n-m, k} \int_{\frac{m+\alpha}{n+\beta+1}}^{\frac{m+\alpha+1}{n+\beta+1}} t^{4} d t \\
& =\frac{1}{5(n+\beta+1)^{4}}\left\{5 n^{4} P_{n, k}^{\left\langle\frac{k}{n}\right\rangle}\left(e_{4} ; x\right)+10(2 \alpha+1) n^{3} P_{n}^{\left\langle\frac{k}{n}\right\rangle}\left(e_{3} ; x\right)\right. \\
& +10\left(3 \alpha^{2}+3 \alpha+1\right) n^{2} P_{n, k}^{\left\langle\frac{k}{n}\right\rangle}\left(e_{2} ; x\right) \\
& \left.+5\left(4 \alpha^{3}+6 \alpha^{2}+4 \alpha+1\right) n P_{n, k}^{\left\langle\frac{k}{n}\right\rangle}\left(e_{1} ; x\right)+\left((\alpha+1)^{5}-\alpha^{5}\right) P_{n, k}^{\left\langle\frac{k}{n}\right\rangle}\left(e_{0} ; x\right)\right\}
\end{aligned}
$$

By substituting the values of $P_{n, k}^{\left\langle\frac{k}{n}\right\rangle}\left(e_{i} ;.\right)(i=0,1,2,3,4)$, we obtain the desired results.

Corollary 2.10. Let $n \in \mathbb{N}, \quad k>0$. Then the central moments of the operators $K_{n}^{(\alpha, \beta, k)}$ are given by

$$
\begin{aligned}
K_{n}^{(\alpha, \beta, k)}\left(e_{1}-x ; x\right) & =\frac{2 \alpha+1}{2(n+\beta+1)}-\frac{\beta+1}{n+\beta+1} x \\
K_{n}^{(\alpha, \beta, k)}\left(\left(e_{1}-x\right)^{2} ; x\right) & =\frac{1}{(n+\beta+1)^{2}}\left\{x(1-x)\left(\frac{n^{2}(k+1)}{n+k}-(\beta+1)^{2}\right)\right. \\
& \left.+(\beta+1)(\beta-2 \alpha) x+\frac{(\alpha+1)^{3}-\alpha^{3}}{3}\right\} .
\end{aligned}
$$


Moreover $K_{n}^{(\alpha, \beta, k)}\left(\left(e_{1}-x\right)^{4} ; x\right)=O\left(\frac{1}{n^{2}}\right)$ as $n \rightarrow \infty$.

Proof. By exploiting the previous results and doing some simple computations, we complete the proof of the corollary.

Lemma 2.11. For $n \in \mathbb{N}$ and $k>0$, we have

$$
K_{n}^{(\alpha, \beta, k)}\left(\left(e_{1}-x\right)^{2} ; x\right) \leq \xi_{n, k}^{\alpha, \beta},
$$

where $\xi_{n, k}^{\alpha, \beta}=\frac{1}{n+\beta+1}\left\{\frac{k+1}{4}+\beta+2 \alpha+\frac{(\alpha+1)^{3}-\alpha^{3}}{3}\right\}$.

Proof. From Corollary 2.10, it follows

$$
\begin{aligned}
K_{n}^{(\alpha, \beta, k)}\left(\left(e_{1}-x\right)^{2} ; x\right) & =\frac{1}{(n+\beta+1)^{2}}\left\{x(1-x)\left(\frac{n^{2}(k+1)}{n+k}-(\beta+1)^{2}\right)\right. \\
& \left.+(\beta+1)(\beta-2 \alpha) x+\frac{(\alpha+1)^{3}-\alpha^{3}}{3}\right\} \\
& \leq \frac{1}{n+\beta+1}\left\{(k+1) x(1-x)+\frac{(\beta+1)}{n+\beta+1}(\beta+2 \alpha) x+\frac{(\alpha+1)^{3}-\alpha^{3}}{3(n+\beta+1)}\right\} \\
& \leq \frac{1}{n+\beta+1}\left\{(k+1) x(1-x)+(\beta+2 \alpha)+\frac{(\alpha+1)^{3}-\alpha^{3}}{3}\right\} \\
& \leq \frac{1}{n+\beta+1}\left\{\frac{k+1}{4}+\beta+2 \alpha+\frac{(\alpha+1)^{3}-\alpha^{3}}{3}\right\} .
\end{aligned}
$$

Corollary 2.12. By using the results in Corollary 2.10, we get the following limit relations

$$
\begin{aligned}
\lim _{n \rightarrow \infty} n K_{n}^{(\alpha, \beta, k)}\left(e_{1}-x ; x\right) & =\alpha+\frac{1}{2}-(\beta+1) x, \\
\lim _{n \rightarrow \infty} n K_{n}^{(\alpha, \beta, k)}\left(\left(e_{1}-x\right)^{2} ; x\right) & =(k+1) x(1-x), \\
\lim _{n \rightarrow \infty} n^{2} K_{n}^{(\alpha, \beta, k)}\left(\left(e_{1}-x\right)^{4} ; x\right) & =3(k+1)^{2} x^{2}(1-x)^{2} .
\end{aligned}
$$

\subsection{Convergence properties of $K_{n}^{(\alpha, \beta, k)}$}

Theorem 2.13. Let $n \in \mathbb{N}, k>0$. Then for every $f \in C[0,1]$, we have

$$
\lim _{n \rightarrow \infty} K_{n}^{(\alpha, \beta, k)}(f ; x)=f(x)
$$

uniformly in $[0,1]$.

Proof. Making use of the results in Lemma 2.9, we deduce that

$$
\lim _{n \rightarrow \infty} K_{n}^{(\alpha, \beta, k)}\left(e_{i}(t) ; x\right)=e_{i}(x) \quad, \quad i=0,1,2
$$

uniformly in $[0,1]$. According to Korovkin's theorem, one can easily get the desired result.

Now, we give the theorems for the rates of convergence of the operators by virtue of the classical modulus of continuity given by (2.2).

Theorem 2.14. Let $n \in \mathbb{N}, k>0$. Then for every $f \in C[0,1]$, we have the following result

$$
\left|K_{n}^{(\alpha, \beta, k)}(f ; x)-f(x)\right| \leq 2 \omega\left(f ; \sqrt{K_{n}^{(\alpha, \beta, k)}\left(\left(e_{1}-x\right)^{2} ; x\right)}\right),
$$

where $\omega(f ;$.$) is modulus of continuity defined by (2.2).$ 
Proof. Taking into consideration [34, p. 1196, Theorem 1], we obtain the desired result easily.

Theorem 2.15. If $f \in C^{1}[0,1]$, then

$$
\left|K_{n}^{(\alpha, \beta, k)}(f ; x)-f(x)\right| \leq\left|\nu_{1}\right|\left|f^{\prime}(x)\right|+2 \sqrt{\nu_{2}} \omega\left(f^{\prime} ; \sqrt{\nu_{2}}\right),
$$

where $\nu_{1}=K_{n}^{(\alpha, \beta, k)}\left(e_{1}-x ; x\right)$ and $\nu_{2}=K_{n}^{(\alpha, \beta, k)}\left(\left(e_{1}-x\right)^{2} ; x\right)$.

Proof. The proof follows directly by applying the result in [7, p. 268, Theorem 5.1.2]. Hence the details are omitted.

Theorem 2.16. Let $f \in C[0,1]$. Then for any $x \in(0,1)$ at which $f^{\prime}, f^{\prime \prime}$ exist, we have $\lim _{n \rightarrow \infty} n\left[K_{n}^{(\alpha, \beta, k)}(f ; x)-f(x)\right]=\frac{1}{2}\left[(2 \alpha+1-2(\beta+1) x) f^{\prime}(x)+(k+1) x(1-x) f^{\prime \prime}(x)\right]$.

Proof. Let $x \in[0,1]$. According to Taylor's series expansion of the function $f$ at the point $x$, we can write

$$
f(s)=f(x)+f^{\prime}(x)(s-x)+\frac{1}{2} f^{\prime \prime}(x)(s-x)^{2}+\eta(s, x)(s-x)^{2},
$$

where $\eta(s, x) \in C[0,1]$ which satisfies

$$
\lim _{s \rightarrow x} \eta(s, x)=0 .
$$

Employing the operators $K_{n}^{(\alpha, \beta, k)}$ to the both sides of (2.12) and taking limit for $n \rightarrow \infty$, it follows that

$$
\begin{aligned}
\lim _{n \rightarrow \infty} n\left(K_{n}^{(\alpha, \beta, k)}(f ; x)-f(x)\right) & =f^{\prime}(x) \lim _{n \rightarrow \infty} n K_{n}^{(\alpha, \beta, k)}(s-x ; x) \\
& +\frac{1}{2} f^{\prime \prime}(x) \lim _{n \rightarrow \infty} n K_{n}^{(\alpha, \beta, k)}\left((s-x)^{2} ; x\right) \\
& +\lim _{n \rightarrow \infty} n K_{n}^{(\alpha, \beta, k)}\left(\eta(s, x)(s-x)^{2} ; x\right) .
\end{aligned}
$$

Applying Cauchy-Schwarz inequality to the last term $n K_{n}^{(\alpha, \beta, k)}\left(\eta(s, x)(s-x)^{2} ; x\right)$, we have

$$
n K_{n}^{(\alpha, \beta, k)}\left(\eta(s, x)(s-x)^{2} ; x\right) \leq \sqrt{K_{n}^{(\alpha, \beta, k)}\left(\eta^{2}(s, x) ; x\right)} \sqrt{n^{2} K_{n}^{(\alpha, \beta, k)}\left((s-x)^{4} ; x\right)} .
$$

Taking into account

$$
\lim _{s \rightarrow x} \eta^{2}(s, x)=0
$$

where $\eta^{2}(s, x) \in C[0,1]$ and according to Theorem 2.13, we can write

$$
\lim _{n \rightarrow \infty} K_{n}^{(\alpha, \beta, k)}\left(\eta^{2}(s, x) ; x\right)=\eta^{2}(x, x)=0 .
$$

Moreover, considering the limit relation which is given by (2.8), we have

$$
\lim _{n \rightarrow \infty} n^{2} K_{n}^{(\alpha, \beta, k)}\left(\left(e_{1}-x\right)^{4} ; x\right)=3(k+1)^{2} x^{2}(1-x)^{2} .
$$

Combining $(2.8),(2.14)$ and $(2.16)$, we obtain the following result

$$
\lim _{n \rightarrow \infty} n K_{n}^{(\alpha, \beta, k)}\left(\eta(s, x)(s-x)^{2} ; x\right)=0 .
$$

Finally, by (2.6) and (2.7) we arrive at

$\lim _{n \rightarrow \infty} n\left(K_{n}^{(\alpha, \beta, k)}(f ; x)-f(x)\right)=\frac{1}{2}\left[(2 \alpha+1-2(\beta+1) x) f^{\prime}(x)+(k+1) x(1-x) f^{\prime \prime}(x)\right]$, which is the required result. 
Remark 2.17. If we get $\alpha=\beta=0$ and $k=1$ in Theorem 2.16, we obtain the Voronovskaja type theorem for the operators $D_{n}^{*\left(\frac{1}{n}\right)}$ given by Agrawal et al. [6].

Now, we continue by analizing the approximation properties of the operators $K_{n}^{(\alpha, \beta, k)}$ with some examples.

Example 2.18. Let $f(x)=x^{3} \sin (4 \pi x), n=50, \alpha=0.2, \beta=0.5$ and $k=0.4$. Behaviours of the approximation for the modified operators $K_{n}^{(\alpha, \beta, k)}$, the operators $D_{n}^{*\left(\frac{1}{n}\right)}$ which were given in [6] and the classical Kantorovich operators $K_{n}$ are illustrated in Figure 4 . We choose the same function in [6] for the better comparison and in Figure 4, one can see that for $k=0.4$, the operators $K_{n}^{(\alpha, \beta, k)}$ provide a better approximation than the operators $D_{n}^{*\left(\frac{1}{n}\right)}$ to the function $f$ but the classical Kantorovich operators $K_{n}$ have better approximation than the others.

Example 2.19. Consider $f(x)=2 x \cos \left(3 \pi x^{3}\right)$ and $k=0.3$. Figure 5 presents the approximation process of the operators $K_{n}^{(\alpha, \beta, k)}$ for the special choices of $n=30,90,150$, $\alpha=2$ and $\beta=3$. It is clearly seen that as the value of $n$ increases, the approximation of the operators $K_{n}^{(\alpha, \beta, k)}$ is getting better.

Example 2.20. Let $f(x)=x^{5}\left(x-\frac{1}{4}\right) \sin (\pi x)$. Figure 6 shows the convergence of $K_{n}^{(\alpha, \beta, k)}$ to the function $f$ for $n=20, k=0.3,0.6,0.9,1.2,1.5$ and $\alpha=\beta=1$. As the value of $k$ decreases towards to zero, the approximation of the operators $K_{n}^{(\alpha, \beta, k)}$ is getting better.

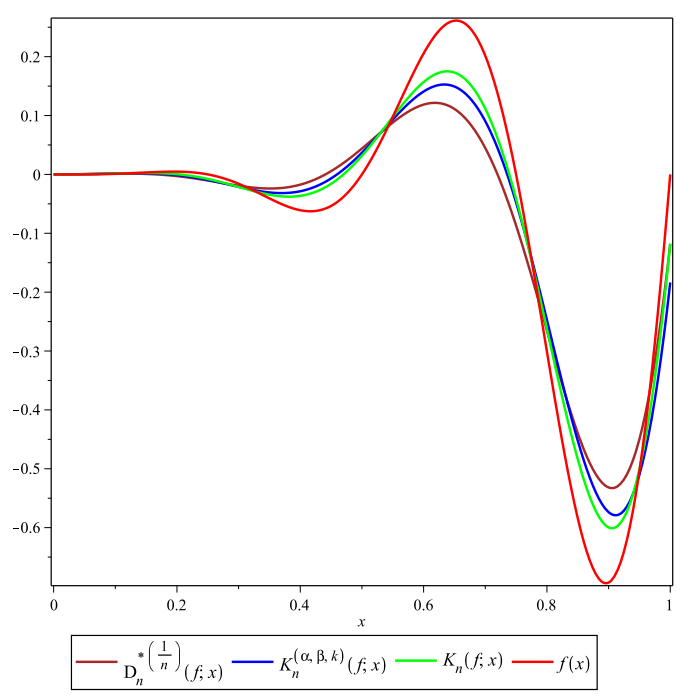

Figure 4. Convergence of $K_{n}^{(\alpha, \beta, k)}, D_{n}^{*\left(\frac{1}{n}\right)}$ and $K_{n}$ to the function $f$ for $n=50$, $\alpha=0.2, \beta=0.5$ and $k=0.4$ 


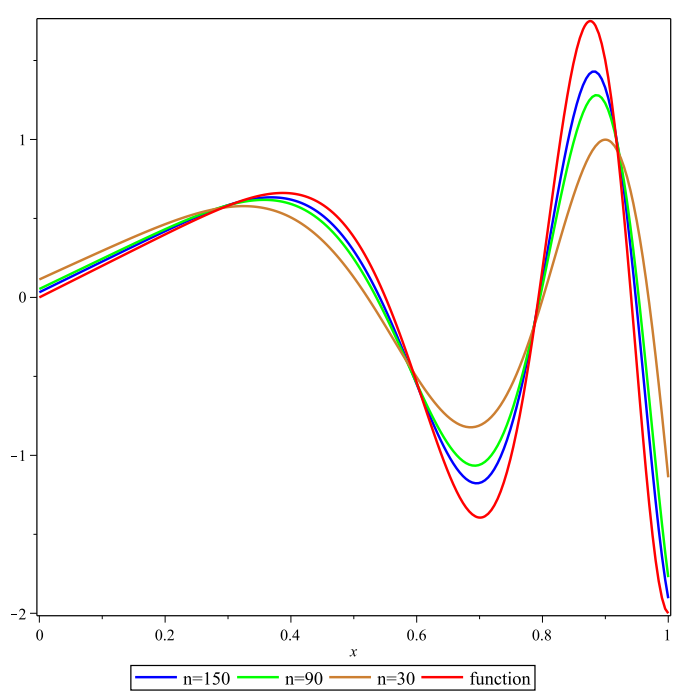

Figure 5. Approximation of the operators $K_{n}^{(\alpha, \beta, k)}$ for $\alpha=2, \beta=3, \quad k=0.3$ and $n=30,90,150$

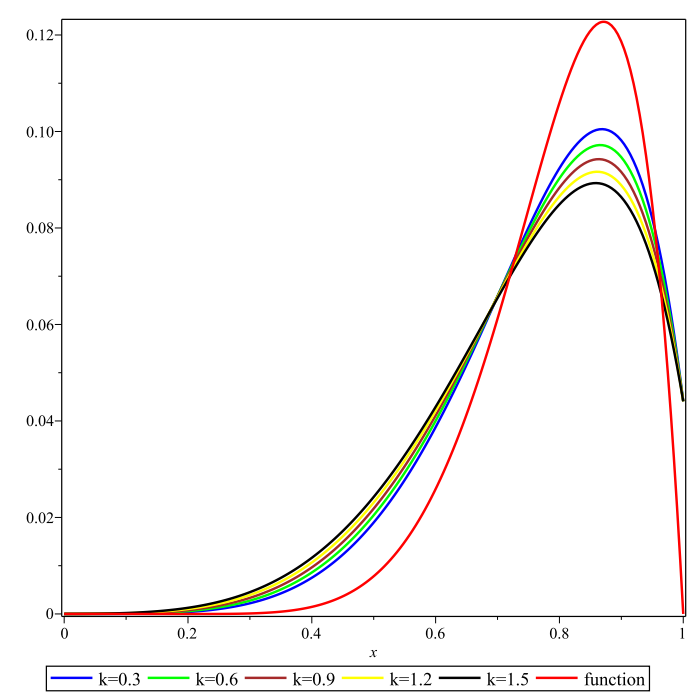

Figure 6. Approximation of the operators $K_{n}^{(\alpha, \beta, k)}$ for $n=20, \alpha=\beta=1$ and $k=0.3,0.6,0.9,1.2,1.5$

\section{Bivariate generalization of the Lupaş-Kantorovich-Stancu type opera- tors by means of Pochhammer $k$-symbol}

In 2016, Agrawal et al. [6] introduced bivariate Kantorovich variant of the operators given by Lupaş and Lupaş [19], and they investigated the rate of convergence by means of the modulus of continuity and proved a Voronovskaja type asymptotic theorem for the bivariate Lupaş-Kantorovich operators. In the same year, Agrawal et al. [5] constructed the bivariate Lupaş-Durrmeyer operators and they examined some approximation properties of these operators using Peetre's K-functional and also discussed the asymptotic behaviour of the operators. In 2018, inspired by the concept of Özarslan and Duman [30], Kajla and Miclaus [14] generalized the Lupaş-Kantorovich type operators based on Polya distribution. They gave the theorem about the degree of approximation and discussed a Voronovskaja type theorem for the bivariate operators. Subsequently, Agrawal and Gupta 
[3] introduced the Kantorovich variant of $q$-analogue of the Stancu operators defined by Nowak [27] and they also studied bivariate operators in their work. Recently, Rahman et al. [31] have presented the bivariate extension of Kantorovich variant of Lupaş operators based on Polya distribution with shifted knots $\alpha_{i}, \beta_{i}, i=1,2$ and have investigated some aproximation properties of these operators.

In this section, we present bivariate Lupaş Kantorovich-Stancu type operators via Pochhammer $k$-symbol mainly motivated by the paper [6]. Also we give rates of convergence via modulus of continuity and the Lipschitz class functions for the bivariate case and we prove a Voronovskaja type theorem for these operators. Finally, we try to illustrate the approximation process with some graphics.

Let $J^{2}=J \times J$ where $J=[0,1]$ and $C\left(J^{2}\right)$ be the space of all real-valued continuous functions on $J^{2}$ endowed with the norm

$$
\|g\|_{C\left(J^{2}\right)}=\sup _{x, y \in J}|g(x, y)|
$$

Let $C^{2}\left(J^{2}\right)$ be the space of all continuous functions $g \in C\left(J^{2}\right)$ such that $\frac{\partial^{i} g}{\partial x^{i}}, \frac{\partial^{i} g}{\partial y^{i}}$ for $i=1,2$ belong to $C\left(J^{2}\right)$. The norm of $g \in C^{2}\left(J^{2}\right)$ is defined by

$$
\|g\|_{C^{2}\left(J^{2}\right)}=\|g\|_{C\left(J^{2}\right)}+\sum_{i=1}^{2}\left(\left\|\frac{\partial^{i} g}{\partial x^{i}}\right\|_{C\left(J^{2}\right)}+\left\|\frac{\partial^{i} g}{\partial y^{i}}\right\|_{C\left(J^{2}\right)}\right)
$$

We construct Lupaş-Kantorovich-Stancu type operators in the bivariate case as follows

$$
\begin{aligned}
& K_{n_{1}, n_{2}}^{\left(\alpha_{1}, \alpha_{2}, \beta_{1}, \beta_{2}, k_{1}, k_{2}\right)}(f ; x, y)=\frac{\left(n_{1}+\beta_{1}+1\right)\left(n_{2}+\beta_{2}+1\right)}{\left(n_{1}\right)_{n_{1}, k_{1}}\left(n_{2}\right)_{n_{2}, k_{2}}} \\
& \times \sum_{m_{1}=0}^{n_{1}} \sum_{m_{2}=0}^{n_{2}}\left(\begin{array}{c}
n_{1} \\
m_{1}
\end{array}\right)\left(\begin{array}{c}
n_{2} \\
m_{2}
\end{array}\right)\left(n_{1} x\right)_{m_{1}, k_{1}}\left(n_{1}-n_{1} x\right)_{n_{1}-m_{1}, k_{1}} \\
& \times\left(n_{2} y\right)_{m_{2}, k_{2}}\left(n_{2}-n_{2} y\right)_{n_{2}-m_{2}, k_{2}} \int_{\frac{m_{1}+\alpha_{1}}{n_{1}+\beta_{1}+1}}^{\frac{m_{1}+\alpha_{1}+1}{n_{1}+\beta_{1}+1}} \int_{\frac{m_{2}+\alpha_{2}}{n_{2}+\beta_{2}+1}}^{\frac{m_{2}+\alpha_{2}+1}{n_{2}+\beta_{2}+1}} f(t, s) d t d s,
\end{aligned}
$$

where $n_{1}, n_{2} \in \mathbb{N}, k_{1}, k_{2}>0$ and $\alpha_{1}, \alpha_{2}, \beta_{1}, \beta_{2}$ are nonnegative real numbers provided $0 \leq \alpha_{1} \leq \alpha_{2} \leq \beta_{1} \leq \beta_{2}$.

For the simplicity we use the notation $K_{n_{1}, n_{2}}^{(\boldsymbol{\alpha}, \boldsymbol{\beta}, \mathbf{k})}$ instead of $K_{n_{1}, n_{2}}^{\left(\alpha_{1}, \alpha_{2}, \beta_{1}, \beta_{2}, k_{1}, k_{2}\right)}$ which is given as

$$
K_{n_{1}, n_{2}}^{\left(\alpha_{1}, \alpha_{2}, \beta_{1}, \beta_{2}, k_{1}, k_{2}\right)}(f ; x, y):=K_{n_{1}, n_{2}}^{(\boldsymbol{\alpha}, \beta, \mathbf{k})}(f ; x, y),
$$

where $\boldsymbol{\alpha}=\left(\alpha_{1}, \alpha_{2}\right), \boldsymbol{\beta}=\left(\beta_{1}, \beta_{2}\right), \mathbf{k}=\left(k_{1}, k_{2}\right)$.

It is obvious that the extension $K_{n_{1}, n_{2}}^{(\boldsymbol{\alpha}, \boldsymbol{k})}$ given above coincides with the operators in [6] when $k_{i}=1$ and $\alpha_{i}=0, \beta_{i}=0$ for $i=1,2$. Also, the special case $\alpha_{2}=\beta_{2}=0$ of the operators in [31] gives the operators $K_{n_{1}, n_{2}}^{(\boldsymbol{\alpha}, \boldsymbol{\beta}, \mathbf{1})}$.

First, we state some basic lemmas which are required to prove approximation properties of $K_{n_{1}, n_{2}}^{(\boldsymbol{\alpha}, \boldsymbol{\beta}, \mathbf{k})}$. Let us denote the monomials $e_{i, j}(x, y)=x^{i} y^{j}$ for $(i, j) \in \mathbb{N}_{0} \times \mathbb{N}_{0}$. 
Lemma 3.1. Let $n_{1}, n_{2} \in \mathbb{N}$ and $k_{1}, k_{2}>0$. From Lemma 2.9, for the operators $K_{n_{1}, n_{2}}^{(\boldsymbol{\alpha}, \boldsymbol{\beta}, \mathbf{k})}$ defined by (3.1), we have

$$
\begin{aligned}
K_{n_{1}, n_{2}}^{(\boldsymbol{\alpha}, \boldsymbol{\beta}, \mathbf{k})}\left(e_{00} ; x, y\right) & =1 \\
K_{n_{1}, n_{2}}^{(\boldsymbol{\alpha}, \boldsymbol{\beta}, \mathbf{k})}\left(e_{10} ; x, y\right) & =\frac{2 \alpha_{1}+1}{2\left(n_{1}+\beta_{1}+1\right)}+\frac{n_{1} x}{n_{1}+\beta_{1}+1}, \\
K_{n_{1}, n_{2}}^{(\boldsymbol{\alpha}, \boldsymbol{\beta}, \mathbf{k})}\left(e_{01} ; x, y\right) & =\frac{2 \alpha_{2}+1}{2\left(n_{2}+\beta_{2}+1\right)}+\frac{n_{2} y}{n_{2}+\beta_{2}+1}, \\
K_{n_{1}, n_{2}}^{(\boldsymbol{\alpha}, \boldsymbol{\beta}, \mathbf{k})}\left(e_{20} ; x, y\right) & =\frac{n_{1}^{2}}{\left(n_{1}+\beta_{1}+1\right)^{2}}\left[x^{2}+x(1-x)\left(\frac{k_{1}+1}{n_{1}+k_{1}}\right)\right] \\
& +\frac{\left(2 \alpha_{1}+1\right) n_{1} x}{\left(n_{1}+\beta_{1}+1\right)^{2}}+\frac{\left(\alpha_{1}+1\right)^{3}-\alpha_{1}^{3}}{3\left(n_{1}+\beta_{1}+1\right)^{2}}, \\
K_{n_{1}, n_{2}}^{(\boldsymbol{\alpha}, \boldsymbol{\beta}, \mathbf{k})}\left(e_{02} ; x, y\right) & =\frac{n_{2}^{2}}{\left(n_{2}+\beta_{2}+1\right)^{2}}\left[y^{2}+y(1-y)\left(\frac{k_{2}+1}{n_{2}+k_{2}}\right)\right] \\
& +\frac{\left(2 \alpha_{2}+1\right) n_{2} y}{\left(n_{2}+\beta_{2}+1\right)^{2}}+\frac{\left(\alpha_{2}+1\right)^{3}-\alpha_{2}^{3}}{3\left(n_{2}+\beta_{2}+1\right)^{2}} .
\end{aligned}
$$

Corollary 3.2. Let $n_{1}, n_{2} \in \mathbb{N}$ and $k_{1}, k_{2}>0$. From Lemma 3.1, the central moments of the operators $K_{n_{1}, n_{2}}^{(\boldsymbol{\alpha}, \boldsymbol{\beta}, \mathbf{k})}$ are given by

$$
\begin{aligned}
K_{n_{1}, n_{2}}^{(\boldsymbol{\alpha}, \boldsymbol{\beta}, \mathbf{k})}\left(e_{10}-x ; x, y\right) & =\frac{2 \alpha_{1}+1}{2\left(n_{1}+\beta_{1}+1\right)}-\frac{\left(\beta_{1}+1\right) x}{n_{1}+\beta_{1}+1}, \\
K_{n_{1}, n_{2}}^{(\boldsymbol{\alpha}, \boldsymbol{\beta}, \mathbf{k})}\left(e_{01}-y ; x, y\right) & =\frac{2 \alpha_{2}+1}{2\left(n_{2}+\beta_{2}+1\right)}-\frac{\left(\beta_{2}+1\right) y}{n_{2}+\beta_{2}+1}, \\
K_{n_{1}, n_{2}}^{(\boldsymbol{\alpha}, \boldsymbol{\beta}, \mathbf{k})}\left(\left(e_{10}-x\right)^{2} ; x, y\right) & =\frac{1}{\left(n_{1}+\beta_{1}+1\right)^{2}}\left\{x(1-x)\left(\frac{n_{1}^{2}\left(k_{1}+1\right)}{n_{1}+k_{1}}-\left(\beta_{1}+1\right)^{2}\right)\right. \\
& \left.+\left(\beta_{1}+1\right)\left(\beta_{1}-2 \alpha_{1}\right) x+\frac{\left(\alpha_{1}+1\right)^{3}-\alpha_{1}^{3}}{3}\right\}, \\
K_{n_{1}, n_{2}}^{(\boldsymbol{\alpha}, \boldsymbol{\beta}, \mathbf{k})}\left(\left(e_{01}-y\right)^{2} ; x, y\right) & =\frac{1}{\left(n_{2}+\beta_{2}+1\right)^{2}}\left\{y(1-y)\left(\frac{n_{2}^{2}\left(k_{2}+1\right)}{n_{2}+k_{2}}-\left(\beta_{2}+1\right)^{2}\right)\right. \\
& \left.+\left(\beta_{2}+1\right)\left(\beta_{2}-2 \alpha_{2}\right) y+\frac{\left(\alpha_{2}+1\right)^{3}-\alpha_{2}^{3}}{3}\right\} .
\end{aligned}
$$

Lemma 3.3. Let $n_{1}, n_{2} \in \mathbb{N}$ and $k_{1}, k_{2}>0$. Then for every $f \in C\left(J^{2}\right)$,

$$
\lim _{n_{1}, n_{2} \rightarrow \infty} K_{n_{1}, n_{2}}^{(\boldsymbol{\alpha}, \boldsymbol{\beta}, \mathbf{k})}\left(e_{i j} ; x, y\right)=e_{i j},
$$

for $(i, j) \in\{(0,0),(0,1),(1,0)\}$ and

$$
\lim _{n_{1}, n_{2} \rightarrow \infty} K_{n_{1}, n_{2}}^{(\boldsymbol{\alpha}, \boldsymbol{\beta}, \mathbf{k})}\left(e_{20}+e_{02} ; x, y\right)=e_{20}+e_{02}
$$

uniformly on $J^{2}, J=[0,1]$.

Theorem 3.4. Let $n_{1}, n_{2} \in \mathbb{N}$ and $k_{1}, k_{2}>0$. Then for every $f \in C\left(J^{2}\right)$, we have

$$
\lim _{n_{1}, n_{2} \rightarrow \infty}\left\|K_{n_{1}, n_{2}}^{(\boldsymbol{\alpha}, \boldsymbol{\beta}, \mathbf{k})}(f)-f\right\|=0 .
$$

Proof. According to the Korovkin's theorem for the bivariate case given in [36], by applying the results given in Lemma 3.3, we get the desired result. 
For any function $f \in C\left(J^{2}\right)$, the complete modulus of continuity for bivariate case is defined as follows:

$$
\widetilde{\omega}(f ; \delta)=\sup \left\{|f(t, s)-f(x, y)|:(t, s),(x, y) \in J^{2} \text { and } \sqrt{(t-x)^{2}+(s-y)^{2}} \leq \delta\right\} .
$$

Moreover, the partial moduli of continuity of $f$ with respect to $x$ and $y$ is given by

$$
\begin{aligned}
& \omega_{1}(f ; \delta)=\sup \left\{\left|f\left(x_{1}, y\right)-f\left(x_{2}, y\right)\right|: y \in J \text { and }\left|x_{1}-x_{2}\right| \leq \delta\right\}, \\
& \omega_{2}(f ; \delta)=\sup \left\{\left|f\left(x, y_{1}\right)-f\left(x, y_{2}\right)\right|: x \in J \text { and }\left|y_{1}-y_{2}\right| \leq \delta\right\} .
\end{aligned}
$$

Theorem 3.5. Let $n_{1}, n_{2} \in \mathbb{N}$ and $k_{1}, k_{2}>0$. Then for every $f \in C\left(J^{2}\right)$ for all $(x, y) \in$ $J^{2}$, we have the following result

$$
\left|K_{n_{1}, n_{2}}^{(\boldsymbol{\alpha}, \boldsymbol{\beta}, \mathbf{k})}(f ; x, y)-f(x, y)\right| \leq 2 \widetilde{\omega}\left(f ; \sqrt{\xi_{n_{1}, k_{1}}^{\left(\alpha_{1}, \beta_{1}\right)}+\xi_{n_{2}, k_{2}}^{\left(\alpha_{2}, \beta_{2}\right)}}\right),
$$

where

$$
\xi_{n_{i}, k_{i}}^{\left(\alpha_{i}, \beta_{i}\right)}=\frac{1}{n_{i}+\beta_{i}+1}\left(\frac{k_{i}+1}{4}+\beta_{i}+2 \alpha_{i}+\frac{\left(\alpha_{i}+1\right)^{3}-\alpha_{i}^{3}}{3}\right), i=1,2
$$

and $\widetilde{\omega}(f ;$.$) is the complete modulus of continuity defined by (3.3).$

Proof. By the definition of the operators (3.1), the complete modulus of continuity (3.3) and Cauchy-Schwarz inequality, it follows

$$
\begin{aligned}
& \left|K_{n_{1}, n_{2}}^{(\boldsymbol{\alpha}, \boldsymbol{\beta}, \mathbf{k})}(f ; x, y)-f(x, y)\right| \\
& \leq K_{n_{1}, n_{2}}^{(\boldsymbol{\alpha}, \boldsymbol{\beta}, \mathbf{k})}(|f(t, s)-f(x, y)| ; x, y) \\
& \leq \widetilde{\omega}\left(f ; \delta_{n_{1}, n_{2}}\right)\left(1+\frac{1}{\delta_{n_{1}, n_{2}}} K_{n_{1}, n_{2}}^{(\boldsymbol{\alpha}, \boldsymbol{\beta}, \mathbf{k})}\left(\sqrt{(t-x)^{2}+(s-y)^{2}} ; x, y\right)\right) \\
& \leq \widetilde{\omega}\left(f ; \delta_{n_{1}, n_{2}}\right)\left(1+\frac{1}{\delta_{n_{1}, n_{2}}}\left(K_{n_{1}, n_{2}}^{(\boldsymbol{\alpha}, \boldsymbol{\beta}, \mathbf{k})}\left((t-x)^{2}+(s-y)^{2} ; x, y\right)\right)^{1 / 2}\right) \\
& \leq \widetilde{\omega}\left(f ; \delta_{n_{1}, n_{2}}\right)\left(1+\frac{1}{\delta_{n_{1}, n_{2}}}\left(K_{n_{1}}^{\left(\alpha_{1}, \beta_{1}, k_{1}\right)}\left((t-x)^{2} ; x\right)+K_{n_{2}}^{\left(\alpha_{2}, \beta_{2}, k_{2}\right)}\left((s-y)^{2} ; y\right)\right)^{1 / 2}\right) .
\end{aligned}
$$

From Lemma 2.11, we find that

$$
\begin{aligned}
& \left|K_{n_{1}, n_{2}}^{(\boldsymbol{\alpha}, \boldsymbol{\beta}, \mathbf{k})}(f ; x, y)-f(x, y)\right| \\
& \leq \widetilde{\omega}\left(f ; \delta_{n_{1}, n_{2}}\right)\left(1+\frac{1}{\delta_{n_{1}, n_{2}}}\left(\xi_{n_{1}, k_{1}}^{\left(\alpha_{1}, \beta_{1}\right)}+\xi_{n_{2}, k_{2}}^{\left(\alpha_{2}, \beta_{2}\right)}\right)^{1 / 2}\right),
\end{aligned}
$$

where

$$
\begin{aligned}
& \xi_{n_{1}, k_{1}}^{\left(\alpha_{1}, \beta_{1}\right)}=\frac{1}{n_{1}+\beta_{1}+1}\left(\frac{k_{1}+1}{4}+\beta_{1}+2 \alpha_{1}+\frac{\left(\alpha_{1}+1\right)^{3}-\alpha_{1}^{3}}{3}\right), \\
& \xi_{n_{2}, k_{2}}^{\left(\alpha_{2}, \beta_{2}\right)}=\frac{1}{n_{2}+\beta_{2}+1}\left(\frac{k_{2}+1}{4}+\beta_{2}+2 \alpha_{2}+\frac{\left(\alpha_{2}+1\right)^{3}-\alpha_{2}^{3}}{3}\right) .
\end{aligned}
$$

Taking

$$
\delta_{n_{1}, n_{2}}=\sqrt{\xi_{n_{1}, k_{1}}^{\left(\alpha_{1}, \beta_{1}\right)}+\xi_{n_{2}, k_{2}}^{\left(\alpha_{2}, \beta_{2}\right)}}
$$

we reach the required result. 
Theorem 3.6. Let $n_{1}, n_{2} \in \mathbb{N}$ and $k_{1}, k_{2}>0$. Then for every $f \in C\left(J^{2}\right)$ for all $(x, y) \in$ $J^{2}$, we have the following result

$$
\left|K_{n_{1}, n_{2}}^{(\boldsymbol{\alpha}, \boldsymbol{\beta}, \mathbf{k})}(f ; x, y)-f(x, y)\right| \leq 2\left(\omega_{1}\left(f ; \sqrt{\xi_{n_{1}, k_{1}}^{\left(\alpha_{1}, \beta_{1}\right)}}\right)+\omega_{2}\left(f ; \sqrt{\xi_{n_{2}, k_{2}}^{\left(\alpha_{2}, \beta_{2}\right)}}\right)\right),
$$

where $\omega_{1}(f ;$.$) and \omega_{2}(f ;$.$) are the partial moduli of continuity of f$ defined by $(3.4)$ and (3.5), respectively and $\xi_{n_{i}, k_{i}}^{\left(\alpha_{i}, \beta_{i}\right)}$ is as in Theorem 3.5.

Proof. From (3.1) and the Cauchy Schwarz inequality, we can easily obtain

$$
\begin{aligned}
& \left|K_{n_{1}, n_{2}}^{(\boldsymbol{\alpha}, \boldsymbol{\beta}, \mathbf{k})}(f ; x, y)-f(x, y)\right| \leq K_{n_{1}, n_{2}}^{(\boldsymbol{\alpha}, \boldsymbol{\beta}, \mathbf{k})}(|f(t, s)-f(x, y)| ; x, y) \\
& \leq K_{n_{1}, n_{2}}^{(\boldsymbol{\alpha}, \boldsymbol{\beta}, \mathbf{k})}(|f(t, s)-f(t, y)| ; x, y)+K_{n_{1}, n_{2}}^{(\boldsymbol{\alpha}, \boldsymbol{\beta}, \mathbf{k})}(|f(t, y)-f(x, y)| ; x, y) \\
& \leq K_{n_{1}, n_{2}}^{(\boldsymbol{\alpha}, \boldsymbol{\beta}, \mathbf{k})}\left(\omega_{1}(f ;|t-x|) ; x, y\right)+K_{n_{1}, n_{2}}^{(\boldsymbol{\alpha}, \boldsymbol{\beta}, \mathbf{k})}\left(\omega_{2}(f ;|s-y|) ; x, y\right) \\
& \leq \omega_{1}\left(f ; \delta_{n_{1}}\right)\left(1+\frac{1}{\delta_{n_{1}}} K_{n_{1}}^{\left(\alpha_{1}, \beta_{1}, k_{1}\right)}(|t-x| ; x)\right) \\
& +\omega_{2}\left(f ; \delta_{n_{2}}\right)\left(1+\frac{1}{\delta_{n_{2}}} K_{n_{2}}^{\left(\alpha_{2}, \beta_{2}, k_{2}\right)}(|s-y| ; y)\right) \\
& \leq \omega_{1}\left(f ; \delta_{n_{1}}\right)\left(1+\frac{1}{\delta_{n_{1}}}\left(K_{n_{1}}^{\left(\alpha_{1}, \beta_{1}, k_{1}\right)}\left((t-x)^{2} ; x\right)\right)^{1 / 2}\right) \\
& +\omega_{2}\left(f ; \delta_{n_{2}}\right)\left(1+\frac{1}{\delta_{n_{2}}}\left(K_{n_{2}}^{\left(\alpha_{2}, \beta_{2}, k_{2}\right)}\left((s-y)^{2} ; y\right)\right)^{1 / 2}\right)
\end{aligned}
$$

From Lemma 2.11,

$$
\begin{aligned}
\left|K_{n_{1}, n_{2}}^{(\boldsymbol{\alpha}, \boldsymbol{\beta}, \mathbf{k})}(f ; x, y)-f(x, y)\right| & \leq \omega_{1}\left(f ; \delta_{n_{1}}\right)\left(1+\frac{1}{\delta_{n_{1}}}\left(\xi_{n_{1}, k_{1}}^{\left(\alpha_{1}, \beta_{1}\right)}\right)^{1 / 2}\right) \\
& +\omega_{2}\left(f ; \delta_{n_{2}}\right)\left(1+\frac{1}{\delta_{n_{2}}}\left(\xi_{n_{2}, k_{2}}^{\left(\alpha_{2}, \beta_{2}\right)}\right)^{1 / 2}\right) .
\end{aligned}
$$

Taking $\delta_{n_{1}}=\sqrt{\xi_{n_{1}, k_{1}}^{\left(\alpha_{1}, \beta_{1}\right)}}$ and $\delta_{n_{2}}=\sqrt{\xi_{n_{2}, k_{2}}^{\left(\alpha_{2}, \beta_{2}\right)}}$, we complete the proof.

We continue by recalling the definition of the Lipschitz class for bivariate function $f$. It is known that a function $f$ belongs to $\operatorname{Lip}_{M}\left(\gamma_{1}, \gamma_{2}\right)$ if it satisfies

$$
|f(t, s)-f(x, y)| \leq M|t-x|^{\gamma_{1}}|s-y|^{\gamma_{2}},
$$

where $M>0,0<\gamma_{1} \leq 1,0<\gamma_{2} \leq 1$. We now prove the rate of convergence for the bivariate operators by virtue of the Lipschitz class.

Theorem 3.7. Let $M>0,0<\gamma_{1} \leq 1,0<\gamma_{2} \leq 1$ and $f \in \operatorname{Lip}_{M}\left(\gamma_{1}, \gamma_{2}\right)$. Then

$$
\left\|K_{n_{1}, n_{2}}^{(\boldsymbol{\alpha}, \boldsymbol{\beta}, \mathbf{k})}(f)-f\right\| \leq M\left(\lambda_{n_{1}, k_{1}}^{\left(\alpha_{1}, \beta_{1}\right)}\right)^{\frac{\gamma_{1}}{2}}\left(\lambda_{n_{2}, k_{2}}^{\left(\alpha_{2}, \beta_{2}\right)}\right)^{\frac{\gamma_{2}}{2}},
$$

where $\lambda_{n_{1}, k_{1}}^{\left(\alpha_{1}, \beta_{1}\right)}=\left\|K_{n_{1}}^{\left(\alpha_{1}, \beta_{1}, k_{1}\right)}\left((t-.)^{2} ;.\right)\right\|$ and $\lambda_{n_{2}, k_{2}}^{\left(\alpha_{2}, \beta_{2}\right)}=\left\|K_{n_{2}}^{\left(\alpha_{2}, \beta_{2}, k_{2}\right)}\left((s-.)^{2} ;.\right)\right\|$.

Proof. Taking into account $f \in \operatorname{Lip}_{M}\left(\gamma_{1}, \gamma_{2}\right)$, this allows us to write

$$
\begin{aligned}
\left|K_{n_{1}, n_{2}}^{(\boldsymbol{\alpha}, \boldsymbol{\beta}, \mathbf{k})}(f ; x, y)-f(x, y)\right| & \leq K_{n_{1}, n_{2}}^{(\boldsymbol{\alpha}, \boldsymbol{\beta}, \mathbf{k})}(|f(t, s)-f(x, y)| ; x, y) \\
& \leq M K_{n_{1}, n_{2}}^{(\boldsymbol{\alpha}, \boldsymbol{\beta}, \mathbf{k})}\left(|t-x|^{\gamma_{1}}|s-y|^{\gamma_{2}} ; x, y\right) \\
& =M K_{n_{1}}^{\left(\alpha_{1}, \beta_{1}, k_{1}\right)}\left(|t-x|^{\gamma_{1}} ; x\right) K_{n_{2}}^{\left(\alpha_{2}, \beta_{2}, k_{2}\right)}\left(|s-y|^{\gamma_{2}} ; y\right) .
\end{aligned}
$$


Applying the Hölder's inequality, we get

$$
\begin{aligned}
\left|K_{n_{1}, n_{2}}^{(\boldsymbol{\alpha}, \beta, \mathbf{k})}(f ; x, y)-f(x, y)\right| & \leq M\left(K_{n_{1}}^{\left(\alpha_{1}, \beta_{1}, k_{1}\right)}\left((t-x)^{2} ; x\right)\right)^{\gamma_{1} / 2}\left(K_{n_{1}}^{\left(\alpha_{1}, \beta_{1}, k_{1}\right)}\left(e_{0} ; x\right)\right)^{\frac{2-\gamma_{1}}{2}} \\
& \times\left(K_{n_{2}}^{\left(\alpha_{2}, \beta_{2}, k_{2}\right)}\left((s-y)^{2} ; y\right)\right)^{\gamma_{2} / 2}\left(K_{n_{2}}^{\left(\alpha_{2}, \beta_{2}, k_{2}\right)}\left(e_{0} ; y\right)\right)^{\frac{2-\gamma_{2}}{2}} \\
& \leq M\left(\lambda_{n_{1}, k_{1}}^{\left(\alpha_{1}, \beta_{1}\right)}\right)^{\frac{\gamma_{1}}{2}}\left(\lambda_{n_{2}, k_{2}}^{\left(\alpha_{2}, \beta_{2}\right)}\right)^{\frac{\gamma_{2}}{2}} .
\end{aligned}
$$

Theorem 3.8. Let $n_{1}, n_{2} \in \mathbb{N}$ and $k_{1}, k_{2}>0$. If $f$ has partial derivatives $f_{x}$ and $f_{y}$ on $J^{2}$, then for all $(x, y) \in J^{2}$, we have

$$
\left\|K_{n_{1}, n_{2}}^{(\boldsymbol{\alpha}, \boldsymbol{\beta}, \mathbf{k})}(f)-f\right\| \leq\left\|f_{x}\right\|_{C\left(J^{2}\right)}\left(\lambda_{n_{1}, k_{1}}^{\left(\alpha_{1}, \beta_{1}\right)}\right)^{\frac{1}{2}}+\left\|f_{y}\right\|_{C\left(J^{2}\right)}\left(\lambda_{n_{2}, k_{2}}^{\left(\alpha_{2}, \beta_{2}\right)}\right)^{\frac{1}{2}}
$$

where $\lambda_{n_{1}, k_{1}}^{\left(\alpha_{1}, \beta_{1}\right)}$ and $\lambda_{n_{2}, k_{2}}^{\left(\alpha_{2}, \beta_{2}\right)}$ are given as in Theorem 3.7.

Proof. Since $f \in C^{1}\left(J^{2}\right)$, we can write as follows

$$
f(t, s)-f(x, y)=\int_{x}^{t} f_{u}(u, s) d u+\int_{y}^{s} f_{v}(x, v) d v .
$$

Applying $K_{n_{1}, n_{2}}^{(\boldsymbol{\alpha}, \boldsymbol{\beta}, \mathbf{k})}$ to the both sides of (3.7), we obtain

$$
\begin{aligned}
& \left|K_{n_{1}, n_{2}}^{(\boldsymbol{\alpha}, \boldsymbol{\beta}, \mathbf{k})}(f ; x, y)-f(x, y)\right| \\
& \leq K_{n_{1}, n_{2}}^{(\boldsymbol{\alpha}, \boldsymbol{\beta}, \mathbf{k})}\left(\left|\int_{x}^{t} f_{u}(u, s) d u\right| ; x, y\right)+K_{n_{1}, n_{2}}^{(\boldsymbol{\alpha}, \boldsymbol{\beta}, \mathbf{k})}\left(\left|\int_{y}^{s} f_{v}(x, v) d v\right| ; x, y\right) .
\end{aligned}
$$

With the help of the inequalities which are given as follows

$$
\left|\int_{x}^{t} f_{u}(u, s) d u\right| \leq\left\|f_{x}\right\|_{C\left(J^{2}\right)}|t-x| \text { and }\left|\int_{y}^{s} f_{v}(x, v) d v\right| \leq\left\|f_{y}\right\|_{C\left(J^{2}\right)}|s-y|,
$$

we can write

$$
\begin{aligned}
& \left|K_{n_{1}, n_{2}}^{(\boldsymbol{\alpha}, \boldsymbol{\beta}, \mathbf{k})}(f ; x, y)-f(x, y)\right| \\
& \leq\left\|f_{x}\right\|_{C\left(J^{2}\right)} K_{n_{1}}^{\left(\alpha_{1}, \beta_{1}, k_{1}\right)}(|t-x| ; x)+\left\|f_{y}\right\|_{C\left(J^{2}\right)} K_{n_{2}}^{\left(\alpha_{2}, \beta_{2}, k_{2}\right)}(|s-y| ; y) .
\end{aligned}
$$

By considering Cauchy Schwarz inequality, from Corollary 3.2, we can deduce the desired result as

$$
\begin{aligned}
\left|K_{n_{1}, n_{2}}^{(\boldsymbol{\alpha}, \boldsymbol{\beta}, \mathbf{k})}(f ; x, y)-f(x, y)\right| & \leq\left\|f_{x}\right\|_{C\left(J^{2}\right)}\left(K_{n_{1}}^{\left(\alpha_{1}, \beta_{1}, k_{1}\right)}\left((t-x)^{2} ; x\right)\right)^{\frac{1}{2}}\left(K_{n_{1}}^{\left(\alpha_{1}, \beta_{1}, k_{1}\right)}\left(e_{0} ; x\right)\right)^{\frac{1}{2}} \\
& +\left\|f_{y}\right\|_{C\left(J^{2}\right)}\left(K_{n_{2}}^{\left(\alpha_{2}, \beta_{2}, k_{2}\right)}\left((s-y)^{2} ; y\right)\right)^{\frac{1}{2}}\left(K_{n_{2}}^{\left(\alpha_{2}, \beta_{2}, k_{2}\right)}\left(e_{0} ; y\right)\right)^{\frac{1}{2}} \\
& \leq\left\|f_{x}\right\|_{C\left(J^{2}\right)}\left(\lambda_{n_{1}, k_{1}}^{\left(\alpha_{1}, \beta_{1}\right)}\right)^{\frac{1}{2}}+\left\|f_{y}\right\|_{C\left(J^{2}\right)}\left(\lambda_{n_{2}, k_{2}}^{\left(\alpha_{2}, \beta_{2}\right)}\right)^{\frac{1}{2}} .
\end{aligned}
$$

Theorem 3.9. For $f \in C^{2}\left(J^{2}\right)$, we have

$$
\begin{aligned}
& \lim _{n \rightarrow \infty} n\left(K_{n, n}^{(\boldsymbol{\alpha}, \boldsymbol{\beta}, \mathbf{k})}(f ; x, y)-f(x, y)\right) \\
& =\left(\alpha_{1}+\frac{1}{2}-\left(\beta_{1}+1\right) x\right) f_{x}(x, y)+\left(\alpha_{2}+\frac{1}{2}-\left(\beta_{2}+1\right) y\right) f_{y}(x, y) \\
& +\frac{1}{2}\left(k_{1}+1\right) x(1-x) f_{x x}(x, y)+\frac{1}{2}\left(k_{2}+1\right) y(1-y) f_{y y}(x, y),
\end{aligned}
$$


uniformly on $J^{2}$.

Proof. Let $(x, y) \in J^{2}$ be arbitrary. In view of the Taylor's series expansion of the function $f$ at the point $(x, y)$, we obtain

$$
\begin{aligned}
f(t, s) & =f(x, y)+f_{x}(x, y)(t-x)+f_{y}(x, y)(s-y) \\
& +\frac{1}{2}\left(f_{x x}(x, y)(t-x)^{2}+2 f_{x y}(x, y)(t-x)(s-y)\right. \\
& \left.+f_{y y}(x, y)(s-y)^{2}\right)+\Omega(t, s ; x, y) \sqrt{(t-x)^{4}+(s-y)^{4}},
\end{aligned}
$$

for $(t, s) \in J^{2}$ where $\Omega(t, s ; x, y) \in C\left(J^{2}\right)$ and $\lim _{(t, s) \rightarrow(x, y)} \Omega(t, s ; x, y)=0$.

Applying the operators $K_{n, n}^{(\boldsymbol{\alpha}, \boldsymbol{\beta}, \mathbf{k})}$ to the both sides of (3.8), it follows

$\lim _{n \rightarrow \infty} n\left(K_{n, n}^{(\boldsymbol{\alpha}, \boldsymbol{\beta}, \mathbf{k})}(f ; x, y)-f(x, y)\right)$

$=f_{x}(x, y) \lim _{n \rightarrow \infty} n K_{n}^{\left(\alpha_{1}, \beta_{1}, k_{1}\right)}((t-x) ; x)+f_{y}(x, y) \lim _{n \rightarrow \infty} n K_{n}^{\left(\alpha_{2}, \beta_{2}, k_{2}\right)}((s-y) ; y)$

$+\frac{1}{2} f_{x x}(x, y) \lim _{n \rightarrow \infty} n K_{n}^{\left(\alpha_{1}, \beta_{1}, k_{1}\right)}\left((t-x)^{2} ; x\right)+f_{x y}(x, y) \lim _{n \rightarrow \infty} n K_{n, n}^{(\boldsymbol{\alpha}, \boldsymbol{\beta}, \mathbf{k})}((t-x)(s-y) ; x, y)$

$+\frac{1}{2} f_{y y}(x, y) \lim _{n \rightarrow \infty} n K_{n}^{\left(\alpha_{2}, \beta_{2}, k_{2}\right)}\left((s-y)^{2} ; y\right)$

$+\lim _{n \rightarrow \infty} n K_{n, n}^{(\boldsymbol{\alpha}, \boldsymbol{\beta}, \mathbf{k})}\left(\Omega(t, s ; x, y) \sqrt{(t-x)^{4}+(s-y)^{4}} ; x, y\right)$.

Using the Cauchy Schwarz inequality to the last term of the right side of the equation (3.9), we reach

$$
\begin{aligned}
& \left(n K_{n, n}^{(\boldsymbol{\alpha}, \boldsymbol{\beta}, \mathbf{k})}\left(\Omega(t, s ; x, y) \sqrt{(t-x)^{4}+(s-y)^{4}} ; x, y\right)\right) \\
& \leq\left(K_{n, n}^{(\boldsymbol{\alpha}, \boldsymbol{\beta}, \mathbf{k})}\left(\Omega^{2}(t, s ; x, y) ; x, y\right)\right)^{\frac{1}{2}}\left(n^{2} K_{n, n}^{(\boldsymbol{\alpha}, \boldsymbol{\beta}, \mathbf{k})}\left((t-x)^{4}+(s-y)^{4} ; x, y\right)\right)^{\frac{1}{2}} \\
& \leq\left(K_{n, n}^{(\boldsymbol{\alpha}, \boldsymbol{\beta}, \mathbf{k})}\left(\Omega^{2}(t, s ; x, y) ; x, y\right)\right)^{\frac{1}{2}}\left(n^{2} K_{n}^{\left(\alpha_{1}, \beta_{1}, k_{1}\right)}\left((t-x)^{4} ; x\right)+n^{2} K_{n}^{\left(\alpha_{2}, \beta_{2}, k_{2}\right)}\left((s-y)^{4} ; y\right)\right)^{\frac{1}{2}} .
\end{aligned}
$$

In view of the Theorem 3.4 , we can write

$$
\lim _{n \rightarrow \infty} K_{n, n}^{(\boldsymbol{\alpha}, \boldsymbol{\beta}, \mathbf{k})}\left(\Omega^{2}(t, s ; x, y) ; x, y\right)=\Omega^{2}(x, y ; x, y)=0
$$

uniformly, since

$$
\lim _{(t, s) \rightarrow(x, y)} \Omega(t, s ; x, y)=0
$$

uniformly on $J^{2}$. Using the equation (3.10) and the limit relations which are given by Corollary 2.12, we have

$$
\lim _{n \rightarrow \infty} n K_{n, n}^{(\boldsymbol{\alpha}, \boldsymbol{\beta}, \mathbf{k})}\left(\Omega(t, s ; x, y) \sqrt{(t-x)^{4}+(s-y)^{4}} ; x, y\right)=0 .
$$

In view of Corollary 2.12 again, we obtain $\lim _{n \rightarrow \infty} n K_{n, n}^{(\boldsymbol{\alpha}, \boldsymbol{\beta}, \mathbf{k})}((t-x)(s-y) ; x, y)=\lim _{n \rightarrow \infty} n K_{n}^{\left(\alpha_{1}, \beta_{1}, k_{1}\right)}(t-x ; x) K_{n}^{\left(\alpha_{2}, \beta_{2}, k_{2}\right)}(s-y ; y)=0$ and we thus find that

$$
\begin{aligned}
& \lim _{n \rightarrow \infty} n\left(K_{n, n}^{(\boldsymbol{\alpha}, \boldsymbol{\beta}, \mathbf{k})}(f ; x, y)-f(x, y)\right) \\
& =\left(\alpha_{1}+\frac{1}{2}-\left(\beta_{1}+1\right) x\right) f_{x}(x, y)+\left(\alpha_{2}+\frac{1}{2}-\left(\beta_{2}+1\right) y\right) f_{y}(x, y) \\
& +\frac{1}{2}\left(k_{1}+1\right) x(1-x) f_{x x}(x, y)+\frac{1}{2}\left(k_{2}+1\right) y(1-y) f_{y y}(x, y),
\end{aligned}
$$


which completes the proof.

Remark 3.10. The case $\alpha_{1}=\alpha_{2}=\beta_{1}=\beta_{2}=0, k_{1}=k_{2}=1$ in Theorem 3.9 presents the Voronovskaja type theorem for the bivariate operators $D_{n, n}^{*\left(\frac{1}{n}, \frac{1}{n}\right)}$ given by Agrawal et al. [6].

Example 3.11. Let $f(x, y)=2 x^{2} y \cos \left(\frac{5 \pi x}{2}\right), n_{1}=n_{2}=20, k_{1}=k_{2}=0.2, \alpha_{1}=\alpha_{2}=$ 0.3 and $\beta_{1}=\beta_{2}=0.5$. The convergence of the bivariate operators $K_{n_{1}, n_{2}}^{(\boldsymbol{\alpha}, \boldsymbol{\beta}, \mathbf{k})}$ (green) and $D_{n_{1}, n_{2}}^{*\left(\frac{1}{n_{1}}, \frac{1}{n_{2}}\right)}$ (yellow) in [6] to the function $f$ (red) is demonstrated in Figure 7. It can be noted that for $k_{1}=k_{2}=0.2$, the approximation of the operators $K_{n_{1}, n_{2}}^{(\boldsymbol{\alpha}, \mathbf{k})}$ is better than the operators $D_{n_{1}, n_{2}}^{*\left(\frac{1}{n_{1}}, \frac{1}{n_{2}}\right)}$ to the function $f$.

Example 3.12. Consider $f(x, y)=2 x \cos (3 \pi(x+y)), k_{1}=k_{2}=0.4, \alpha_{1}=\alpha_{2}=0.3$ and $\beta_{1}=\beta_{2}=0.6$. Figure 8 presents the approximation process of the bivariate operators $K_{n_{1}, n_{2}}^{(\boldsymbol{\alpha}, \boldsymbol{\beta}, \mathbf{k})}$ to the function $f$ (red) for $n_{1}=n_{2}=10,20,40$ (yellow, green, blue, respectively). It is clearly seen that as the values of $n_{1}, n_{2}$ increase, the approximation of the operators $K_{n_{1}, n_{2}}^{(\boldsymbol{\alpha}, \boldsymbol{\beta}, \mathbf{k})}$ is getting better.

Example 3.13. Let $f(x, y)=7 x^{5}\left(x-\frac{1}{4}\right) \sin (2 \pi y)$ (red). Figure 9 shows the convergence of $K_{n_{1}, n_{2}}^{(\boldsymbol{\alpha}, \boldsymbol{\beta}, \mathbf{k})}$ to the function $f$ for $n_{1}=n_{2}=10, \alpha_{1}=\alpha_{2}=\beta_{1}=\beta_{2}=0$ and $k_{1}=k_{2}=$ $0.3,0.9,1.2$ (yellow, green, blue, respectively). As the values of $k_{1}, k_{2}$ decrease towards to zero, the approximation of the bivariate operators $K_{n_{1}, n_{2}}^{(\boldsymbol{\alpha}, \boldsymbol{\beta}, \mathbf{k})}$ is getting better.

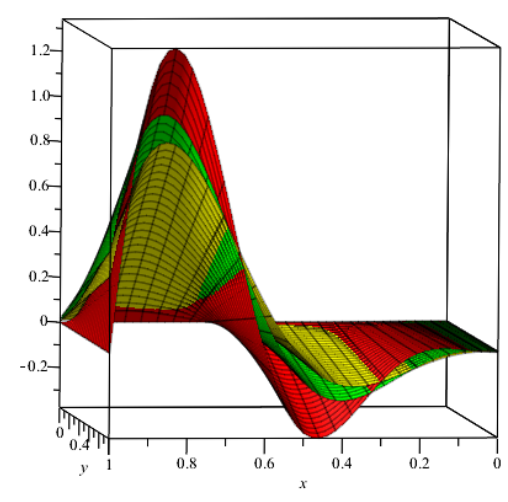

Figure 7. Convergence of $K_{n_{1}, n_{2}}^{(\boldsymbol{\alpha}, \boldsymbol{\beta}, \mathbf{k})}$ and $D_{n_{1}, n_{2}}^{*\left(\frac{1}{n_{1}}, \frac{1}{n_{2}}\right)}$ to the function $f$ 


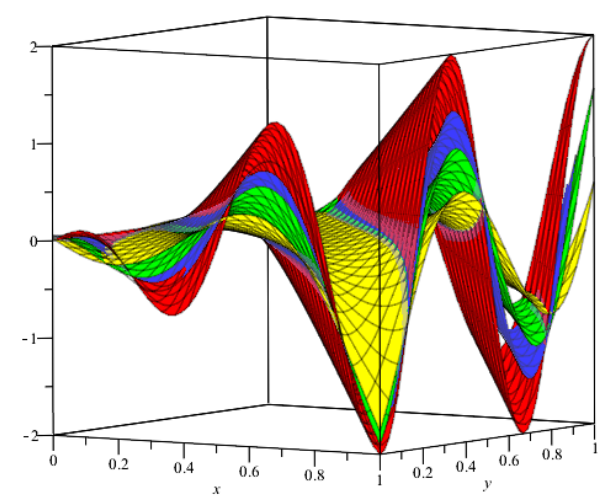

Figure 8. Approximation of the operators $K_{n_{1}, n_{2}}^{(\boldsymbol{\alpha}, \boldsymbol{\beta}, \mathbf{k})}$ for $n_{1}=n_{2}=10,20,40$

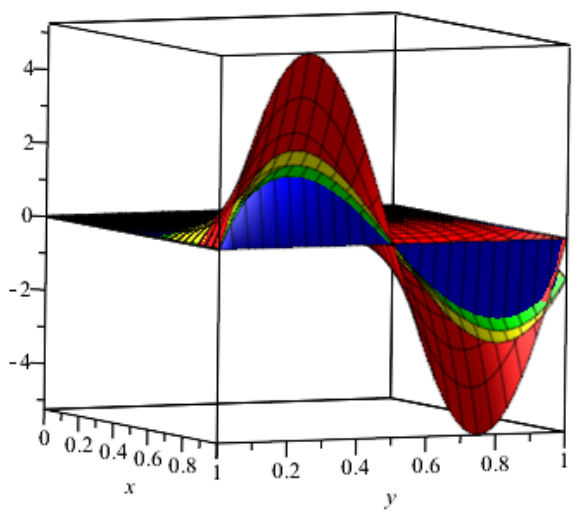

Figure 9. Approximation of the operators $K_{n_{1}, n_{2}}^{(\boldsymbol{\alpha}, \boldsymbol{\beta}, \mathbf{k})}$ for $k_{1}, k_{2}=0.3,0.9,1.2$

Acknowledgment. This work of the first, second and fourth authors was supported by Scientific Research Projects Coordination Unit of Kırıkkale University. Project number 2020/045.

\section{References}

[1] A.M. Acu and H. Gonska, Perturbed Bernstein-type operators, Anal. Math. Phys. 10 (4), 1-26, 2020.

[2] A.M. Acu, N. Manav and D.F. Sofonea, Approximation properties of $\lambda$-Kantorovich operators, J. Inequal. Appl. 2018, 1-12, 2018.

[3] P.N. Agrawal and P. Gupta, q-Lupaş Kantorovich operators based on Pólya distribution, Ann. Univ. Ferrara 64, 1-23, 2018.

[4] P.N. Agrawal, N. Ispir and A. Kajla, Approximation properties of Bézier-summation integral type operators based on Pólya-Bernstein functions, Appl. Math. Comput. 259, 533-539, 2015. 
[5] P.N. Agrawal, N. Ispir and A. Kajla, GBS Operators of Lupaş-Durrmeyer type based on Pólya Distribution, Results Math. 69 (3-4), 397-418, 2016.

[6] P.N. Agrawal, N. Ispir and A. Kajla, Approximation properties of Lupas-Kantorovich operators based on Polya distribution, Rend. Circ. Mat. Palermo 65, 185-208, 2016.

[7] F. Altomare and M. Campiti, Korovkin-type Approximation Theory and its Applications, de Gruyter Studies in Mathematics 17, Walter de Gruyter, Berlin, 1994.

[8] D. Barbosu, Kantorovich Stancu type operators, J. Inequal.Pure Appl. Math. 5 (3), 2004.

[9] S.N. Bernstein, Demonstration du theoreme de Weierstrass Fondee sur le calcul des probabilites, Comp. Comm. Soc. Mat. Charkow Ser. 13 (2), 1-2, 1912.

[10] D. Cárdenas-Morales and V. Gupta, Two families of Bernstein-Durrmeyer type operators, Appl. Math. Comput. 248, 342-353, 2014.

[11] N. Deo, M. Dhamija and D. Miclăuş, Stancu-Kantorovich operators based on inverse Pólya-Eggenberger distribution, Appl. Math. Comput. 273, 281-289, 2016.

[12] R. Diaz and E. Pariguan, On hypergeometric functions and Pochhammer k-symbol, Divulg. Mat. 15 (2), 179-192, 2007.

[13] V. Gupta and T. Rassias, Lupaş-Durrmeyer operators based on Pólya distribution, Banach J. Math. Anal. 8 (2), 146-155, 2014.

[14] A. Kajla and D. Miclăuş, Some smoothness properties of the Lupaş-Kantorovich type operators based on Pólya distribution, Filomat 32 (11), 3867-3880, 2018.

[15] A. Kajla and D. Miclăuş, Approximation by Stancu-Durrmeyer type operators based on Pólya-Eggenberger distribution, Filomat 32 (12), 4249-4261, 2018.

[16] C.G. Kokologiannaki, Properties and inequalities of generalized k-gamma, beta and zeta functions, Int. J. Contemp. Math. Sciences 5 (14), 653-660, 2010.

[17] V. Krasniqi, A limit for the $k$-Gamma and $k$-Beta Function, Int. Math. Forum 5 (33), 2010.

[18] S.F. Li and Y. Dong, k-Hypergeometric series solutions to one type of nonhomogeneous k-hypergeometric equations, Symmetry 11, 262, 2019.

[19] L. Lupaş and A. Lupaş, Polynomials of binomial type and approximation operators, Stud. Univ. Babes-Bolyai Math. 32 (4), 61-69, 1987.

[20] D. Miclăus, The revision of some results for Bernstein Stancu type operators, Carpathian J. Math 28 (2), 289-300, 2012.

[21] D. Miclăuş, On the monotonicity property for the sequence of Stancu type polynomials, An. Ştiint. Univ. Al. I. Cuza Iaşi, Mat. (N.S) 62 (1), 141-149, 2016.

[22] D. Miclăuş, On the Stancu type bivariate approximation formula, Carpathian J. Math. 32 (1), 103-111, 2016.

[23] S.A. Mohiuddine and F. Özger, Approximation of functions by Stancu variant of Bernstein Kantorovich operators based on shape parameter $\alpha$, Rev. R. Acad. Cienc. Exactas. Fis. Nat. Ser. A Math. RACSAM 114 (70), 2020.

[24] S. Mubeen, $k$-Analogue of Kummer's first formula, J. Inequal. Spec. Funct. 3 (3), 41-44, 2012.

[25] S. Mubeen and A. Rehman, A note on k-gamma function and pochhammer $k$-symbol, J. Inform. Math. Sci. 6 (2), 93-107, 2014.

[26] T. Neer and P.N. Agrawal, A genuine family of Bernstein-Durrmeyer type operators based on Pólya basis functions, Filomat, 31 (9), 2611-2623, 2017.

[27] G. Nowak, Approximation properties for generalized q-Bernstein polynomials, J. Math. Anal. Appl. 350, 50-55, 2009.

[28] A.A. Opriş, Approximation by modified Kantorovich Stancu operators, J. Inequal. Appl. 2018 (346), 2018.

[29] S. Ostrovska and M. Turan, The distance between two limit q-Bernstein operators, Rocky Mountain J. Math. 50 (3), 1085-1096, 2020. 
[30] M.A. Özarslan and O. Duman, Smoothness properties of modified BernsteinKantorovich operators, Numer. Funct. Anal. Optim. 37, 92-105, 2016.

[31] S. Rahman, M. Mursaleen and A. Khan, A Kantorovich variant of Lupas-Stancu operators based on Pólya distribution with error estimation, Rev. R. Acad. Cienc. Exactas Fis. Nat. Ser. A Mat. RACSAM 114 (75), 2020.

[32] Q. Razi, Approximation of functions by Bernstein type operators, Master Thesis, Aligarh Muslim University, Aligarh, India, 1983.

[33] Q. Razi, Approximation of a function by Kantorovich type operators, Mat. Vesnik 41, 183-192, 1989.

[34] O. Shisha and B. Mond, The degree of convergence of sequences of linear positive operators, Proc. Natl. Acad. Sci. USA, 60, 1196-1200, 1968.

[35] D.D. Stancu, Approximation of functions by a new class of linear polynomial operators, Rev. Roumaine Math. Pures Appl. 13 (8), 1173-1194, 1968.

[36] V.I. Volkov, On the convergence of sequences of linear positive operators in the space of two variables, Dokl. Akad. Nauk. SSSR (N.S.), 115, 17-19, 1957. 